\title{
The Mechanics of Conceptual Thinking
}

\author{
André Michaud \\ Service de Recherche Pédagogique, Québec, Canada \\ Email: srp2@srpinc.org
}

How to cite this paper: Michaud, A. (2019). The Mechanics of Conceptual Thinking Creative Education, 10, 353-406. https://doi.org/10.4236/ce.2019.102028

Received: January 9, 2019

Accepted: February 18, 2019

Published: February 21, 2019

Copyright (c) 2019 by author(s) and Scientific Research Publishing Inc. This work is licensed under the Creative Commons Attribution International License (CC BY 4.0).

http://creativecommons.org/licenses/by/4.0/

Open Access

\begin{abstract}
Description of the mechanics of conceptual thinking that stems from interaction sequences between the limbic system and the verbal areas of the neocortex. Description of the rise of the attention level to full active awareness when a feeling of uneasiness due to a verbal stimulus is triggered by the amygdala, followed by an active cogitation process involving the verbal areas of the neocortex, ending in the strengthening in the neocortex by the hippocampus of a synaptic network corresponding to a modified verbal sequence that removes or reduces the feeling of uneasiness that initiated the sequence. Description of the generalization ability that emerges from the use of articulated languages, acquired by education, from which conceptual thinking and also the collectively intelligible mathematical language emerge that also develops to various degrees in some individuals by education. Description of the mathematical thinking mode, about whose engrams have been located in the neocortex in areas that do not overlap the verbal areas.
\end{abstract}

\section{Keywords}

Neocortex, Amygdala, Hippocampus, Verbal Areas, Conceptual Thinking, Articulated Verbal Language, Mathematical Nonverbal Language

\section{Introduction}

The knowledge accumulated about the implication of the inner structures of the limbic system in articulated language processing, from which conceptual thinking emerges, is now advanced to the point that a clear mechanics of conceptual thinking can now be formulated. Unexpectedly, it appears that the response reflexes related to fear reactions induced by feelings of danger, controlled by the limbic system, of which the amygdala and the hippocampus are sub-components, are involved in the mechanics of conceptual thinking.

It is indeed in the limbic system, that Paul Chauchard identifies as being the seat of "bio-awareness" (Chauchard, 1963a), that the whole collection of biolog- 
ical signals coming from the senses and the rest of the body is integrated before being put at the disposal of a person's active awareness through the filter of feelings of well being or discomfort. What is perceived as fulfilling organic needs is interpreted as pleasing, and what is perceived as dangerous is interpreted as unpleasant.

"There is here a particularly well developed aspect of bio-awareness that will show its importance by the fact that the neocortex draws from it critical information required to reach true awareness, and the means to ground it in existential and organic reality. If we are not for ourselves an object observable with cold reason, but a living and sentient reality, we owe it first and foremost to this hypothalamic integration" (Chauchard, 1963a: p. 63).

It turns out that the use of articulated language has been found to activate these limbic substructures in a manner that initiates and maintains permanent attentive awareness during waking states in human beings, by constantly drawing the attention of the individual to sequences of nonverbal memories that he has related to these verbal stimuli.

Once activated, these sequences of memories can then be correlated into new configurations that in turn conjure up other sequences of memories in a continuous process that can remain unguided as when daydreaming, or that can be voluntarily oriented in search of specific memories that the subject wishes to correlate in a novel manner with the set of activated memories

At the general level, the biological machinery sustaining conceptual thinking has been identified as mainly involving three cerebral structures that are being deeply studied that is the amygdala, the verbal areas of the neocortex and the hippocampus. These three structures have been observed to activate in a very specific sequence whenever a verbal stimulus generates the slightest feeling of discomfort in the amygdala, causing the individual to pay active attention to what this verbal stimulus reminds him of.

The key role played in conceptual thinking by verbal stimuli is due to the human ability to use an articulated language, which allows us to name absolutely all aspects of all nonverbal objects, processes or events that our senses allow us to perceive, of all nonverbal abstract concepts that we conceive of, as well as of all nonverbal emotions and feelings originating in the limbic system.

Using these names to think about this collection of non-verbal memories leads to the perception of similarities or differences in these sets, resulting in generalizations from which a collection of the generalized non-verbal abstracts concepts emerges that allows us to organize and structure our non-verbal memories in a manner that leads to understanding our environment to a level infinitely superior to any level that can be reached by the animal species not possessing our ability to use an articulated language.

At the functional level, conceptual thinking involves the organisation and manipulation of synaptic imprints located in the verbal areas, generally referred to as "engrams", each engram corresponding to a network of interrelated synapses 
corresponding to a word, itself synaptically related to non-verbal information stored in other areas of the neocortex as other sets of extended networks of synaptic imprints, each non-verbal synaptic imprint corresponding to what the individual recalls of a specific non-verbal object, event, chain of events, abstract concept or emotion, and characteristics thereof.

To simplify the description, the word "object" will often be used in this article in its extended meaning to represent the longer phrase "any object, characteristic, move, process, event, emotion, abstract concept, concrete or abstract image of any nature, etc.", that is, any element of information that can be identified separately from all others.

Generally speaking, the non-verbal information that each word refers to in context of any idea being expressed has been found to be stored in the non-verbal hemisphere of the brain for imprints resulting from the perceptions of the senses and in non-verbal areas of the verbal hemisphere for imprints corresponding to the non-verbal concepts generated by the generalization process that emerges as a consequence of using an articulated language.

The nonverbal "images" of objects, events and processes that the individual becomes aware of originating from the environment reach his active awareness though the filter of the emotions that they elicit during their transit through the limbic system after having been synthesized into coherent representations by specialized multilayer neural networks from the continuous flow of countless signals picked up by the sensitive nerves endings.

"It is only of this coordinated set that we are aware. We do not know the detail of all the incoming messages, but only of the global interpretation, which is the only thing that matters. The primary cerebral interpretation tasks are unconscious" (Chauchard, 1963b: p. 59).

In his essay titled "Versuch einer psychologischen Interpretation der Symptomatologie der Hysterie. L'Encéphale 28, No. 4, 285-293, 1933” (Pickenhain, 1998: p. 250), Ivan Pavlov identified as "the first system of signalization" the set of nonverbal images originating from the environment and the limbic system; that is, a signalization system of the same nature as the less performing thinking mode of species having developed less advanced nervous systems, and identifies as a "second system of signalization" the cerebral structures created by the kinesthetic excitation induced by the use of the speech organs, resulting in the establishment of articulated languages, that bring to the attention of individuals the signals of the first system. He finally concluded that this second system of signalization is "the very last and particularly delicate push of the evolutionary process" (Pickenhain, 1998; Michaud, 2016b; Chauchard, 1960b).

"It was Pavlov who showed that language was a consequence of the human cerebral complexity and that it objectified the superiority and specificity of the human brain with respect to animal brains. He perceived language as a special type of conditioned reflexes, a second system of signalization, the first one being that of gnosis and praxis of direct thinking by images. To each image will be 
substituted through education its verbal denomination. Since they name everything, instead of associating images, human beings can directly associate the corresponding names, a system more efficient in maximizing the abstraction capabilities of the human brain" (Chauchard, 1960b: p. 122).

His conclusion thus was that the ability to acquire articulated language is the outcome of neural activity specific to human beings, involving processes that allow simultaneously abstracting and generalizing the countless signals of the first system in a manner that provides them with the ability to analyze and synthesize this new type of signals, that the words of articulated languages happen to be, into a set of generalized non-verbal abstract concepts that establishes an internal representation of our environment, and that constitutes a new principle allowing boundless orientation in this environment and which is the foundation of humankind's ultimate realizations, that is, science in all of its general empirical forms as well as in its specialized forms (Pickenhain, 1998; Michaud, 2016b).

Here is a quote from the original text from his 1936 essay, for conformity validation, summarizing his conclusions regarding the emergence in human beings of language as a second system of signalization, followed by its translation:

"Damit wird ein neues Prinzip in die Nerventätigkeit eingeführt, die Abstraktion und gleichzeitig damit die Verallgemeinerung der unzähligen Signale des vorhergehenden Systems. Parallel dazu erfolgt ebenfalls das Analysieren und Synthetisieren dieser neuen verallgemeinerten Signale. Dieses Prinzip erlaubt eine grenzenlose Orientierung in der Umwelt und begründet die höchste Anpassung des Menschen, die Wissenschaft, sowohl in Form des allgemeinmenschlichen Empirismus als auch in ihrer spezialisierten Form. Dieses zweite Signalsystem und sein Organ müssen als die allerletzte Errungenschaft des Evolutionsprozesses besonders anfällig sein" (Pickenhain, 1998: p. 265).

"A new principle of nervous activity thus comes into being that simultaneously abstracts and generalizes the countless signals of the first system. These newly generalized signals then become available for subsequent analysis and synthesis. This development allows a limitless orientation in the environment and is the foundation of the highest realization of man, science, in its general empirical forms as well as in its specialized forms. This second system of signalization and its organ thus constitute the very last and particularly delicate progress of the evolutionary process".

The whole collection of synaptic interconnections that articulated languages establish between the various aspects of our nonverbal recollections constitutes a hierarchical structure associative by inclusion that provides us with an easy and immediate access to any subset of the subjective model of reality that each of us elaborates from childhood. The evocation of each word momentarily strengthens the synaptic interconnections that associate this word to various aspects of memories that were connected to this verbal stimulus in the past, in context of the idea in process of being expressed.

Besides organizing and structuring the non-verbal observations originating 
from the senses, and the emotions and sensations originating from the limbic system, into this interconnected structure which is easy to "visit", so to speak, the names allow their generalization, which leads to the establishment of a set of supplementary nonverbal symbolic concepts originating from the use of language, which is as easy to use as the words themselves to think about the events, quantities, shapes and volumes of objects perceived in the environment and of the various processes into which they are involved, providing human beings with the ability to spatially and temporally orient himself in their environment.

Over the course of history, a special subset of these idealized abstract concepts emerged from the use of language as humans exchanged ideas, that is, the idealized geometric concepts resulting from the perceptions of shapes, and the abstract mathematical concepts resulting from the measurement of quantities and processes, and developed into a stable common nonverbal language allowing discussing and exchanging generally useful information about the objects and processes observed in the environment.

This special set of idealized geometric and mathematic concepts, that numerous individuals learn to master to the point of becoming able to directly use in conceptually thinking nonverbally about the processes that are suspected to objectively exist in our environment, will be discussed in the last section of this article. But we will for the moment deal only with the first level of these abstract concepts that emerge directly from the use of articulated language, and from which emerges the whole set of modern idealized geometric and mathematic concepts.

The first level of geometric and mathematic symbolic concepts has been observed to naturally appear in all children as early as 6 months of age in relation with the development of the "the number sense", which is considered the foundation on which all later mathematical abilities develop (Amalric \& Dehaene, 2016). These first abstract mathematical concepts are related to the awaking awareness of the plurality of similar objects, which is at the origin of the expression "the number sense" to name this fledgling ability.

The further mathematical abilities required to manipulate these numbers and geometric concepts, such as addition, subtraction, multiplication and division are typically acquired through elementary schooling; through high school training for third and some higher step abstractions such as basic algebra, geometry, and mathematical analysis; and to the full range of the currently developed steps of higher mathematical concepts for those who take interest.

Conclusive exploratory tests by means of functional magnetic resonance imaging (fMRI) now confirm that the areas of the neocortex where these nonverbal mathematical synaptic imprints are stored lie outside the areas dealing with verbal processing, and that they do not overlap the verbal areas (Amalric \& Dehaene, 2016).

Moreover, it was observed during these conclusive experiments carried out by Marie Amalric and Stanislas Dehaene in 2016, that although the verbal areas of 
non-mathematicians remain fully active during all types of high level cogitation, the verbal areas in the brains of high level mathematicians become less active to the benefit of the mathematical areas even for non-mathematical activities, such as face recognition (Amalric \& Dehaene, 2016), which means that the nonverbal mathematical symbolic engrams that develop in these areas are directly used as a complementary means of cogitation for those who acquire them.

Figure 2 of reference (Amalric \& Dehaene, 2016) identifies the non-verbal areas in both neocortex hemispheres that activate in voluntary subjects mastering high level mathematics. For this reason, and for a second ontological reason that will be put to light further on, the nonverbal mathematical language that develops in these separate neocortex areas will be referred to in this study as the "third system of signalization". Chauchard was already well aware that mathematical thinking is a separate form of signalization:

"The grammatical relations, that is, the manner in which we associate the words of languages is a functional modality of the human grain; from this perspective the progress of languages is grounded on a better usage of the capabilities of the human brain, mathematical symbolism being itself only a sort of language, thus of cerebral signalization" (Chauchard, 1960b: p. 123).

A major observation made during the Amalric-Dehaene experiments was that all domains of high level mathematics that were tested activated the same network of nonverbal brain areas that were already known to activate from early childhood in relation with the emergence of the number sense.

A currently popular hypothesis is that the acquisition of the number sense predates articulated language acquisition, because it was conclusively observed in infants as young as 6 months of age, that is, long before children can speak, that led to an ongoing search for still unknown preverbal ontological evolutionary foundations that could logically explain the emergence of number sense in infants (Starr, Libertus, \& Brannon, 2013).

To this hypothesis however comes in opposition another one shared by Noam Chomsky according to which the number sense would rather be an outcome of the first comprehension acquisition at the level of the articulated language used by the adults who care for the child (Chomsky, 2006), which would establish the acquisition of articulated language as the ontological foundation of the subsequent acquisition of the non-verbal mathematical abilities, and by extension, of all other types of nonverbal symbolic representations not directly originating from sensory or limbic perceptions.

The arguments formulated in favour of the preverbal foundation are not clear however as to when during infancy language acquisition is deemed to begin to be established. It is well understood in this regard that even if children begin to speak on average between 18 and 24 months of age, they understand much sooner the meaning of a number of words and sentences.

The fact is that infants as young as 1 year of age already purposefully point a finger towards new objects that they obviously wish to be told the name of, as 
confirmed by conclusive studies carried out at the Max Planck Institute, which is quite revealing in this regard, and also reveals that this ability to gesturally ask the name of an object can only be the end result of a process towards articulated language acquisition that began even earlier.

How much earlier? Recent research carried out by Erika Bergelson and Daniel Swingley (Bergelson \& Swingley, 2012) confirm that children as young as 6 months old already understand the meaning of a number of spoken words, which interestingly leads to observe that the number sense observed in infants at this age may factually not be preverbal, and that it is quite possible that it could emerge from a verbal foundations.

"Most children do not say their first words until around their first birthday. Nonetheless, infants know some aspects of their language's sound structure by 6 12 month: they learn to perceive their native language's consonant and vowel categories, they recognize the auditory form of frequent words, and they employ these stored word forms to draw generalizations about the sound patterns of their language, using cognitive capacities for pattern finding" (Bergelson \& Swingley, 2012).

Indeed, considering that from the moment that a child understands that many objects in his environment are named with the same word, "chair" for example, it would even be surprising that the idea of a plurality of objects visually identical having the same name would not awaken in his mind the idea of a plurality of objects similar in appearance, all identified with a single word; which would quite naturally explain that as young as 6 months of age, following this first verbal generalization, the child would develop the number sense as observed when the Starr-Libertus-Brannon study (Starr, Libertus, \& Brannon, 2013) is correlated with the Bergelson-Swingley study (Bergelson \& Swingley, 2012).

In reality, it would even seem in relation with this inescapable first possible generalization occurrence, and of its inescapable outcome, that the appearance of the generalization ability and of the number sense would be a single event that could not possibly have ontologically occurred before the establishment of the verbal thinking mode. The correlation between the emergence of the number sense and the appearance of the generalization ability will be analyzed further on.

\section{Overview of the Process of Conceptual Thinking}

Before proceeding to the analysis of the fundamental generalization ability, let us first examine the interactions that have been observed between the verbal areas of the neocortex and the limbic system.

We will first analyze how a particular type of verbal stimuli, originating either from internal cogitation or heard or read from some external source, can over-activate the level of attention to the level of active awareness with respect to other verbal stimuli, coinciding with the triggering a now well identified sequence of amygdala-neocortex-hippocampus activation, that causes the individual to pay more acutely attention to the set of memories that this particular ver- 
bal stimulus conjures up. This momentarily more acute level of awareness about this set of activated elements then allows the subject to identify the specific aspect of the set that triggered his heightened level of attention.

If this identification results in a refocusing of his understanding of the set in a way that pleases the subject sufficiently for him to be satisfied with the new configuration, it is observed that the hippocampus will automatically strengthen the set of synaptic links associating the engrams considered into this new configuration.

However, if no satisfying refocusing shortly occurs, the subject may lose interest if another verbal stimulus causes the amygdala to strengthen a different set of engrams to the level of active awareness, which will result in the unresolved issue being inhibited out of the field of active awareness until some other verbal stimulus re-activates it.

Due to the permanent involvement of the limbic system during the conceptual thinking process, absolutely all aspects of human being's cogitations are de facto considered through the subjective filter of the emotional reactions generated in the limbic system while this information was initially recorded, as well as through the filter presumably different of the emotional reactions of the individual each time this information is being attentively remembered and reconsidered, which means that any objective reconsideration should ideally take into account the existence of these two separate subjective emotional filters.

This emotional response is due to the fact that the limbic system, to which the amygdala and the hippocampus belong, is genetically designed to primarily attend to the triggering of immediate instinctive protection reactions when subjects feel any impression of danger, real or imagined, in the information coming from their senses.

It is well established that in mammals, the limbic system is the seat of all emotions. It was experimentally demonstrated in the first half of the $20^{\text {th }}$ century, that by electrically stimulating the hippocampus of mammals such as cats, reactions of fear, rage, attack, flight and defensive behavior are elicited, that "bring the animal to the highest levels of alertness, and prepare it for the most extreme levels of physical action that may be necessary to ensure its survival' (Shepherd, 1994: p. 607).

Since the level of danger felt by the subject determines the intensity of the amygdala response, in case of a feeling of extreme danger, this highest level of intensity triggers the immediate choice by the hippocampus of the reaction that instinctively seems the most likely to save the subject's life at this particular moment, that is, either fight or flight, that is, an invariant pattern of instinctive behavioral reactions so well identified and documented that it was formally named the fight-or-fight response.

If we now define the feeling of "fear" as a feeling of "extreme uneasiness" that raises the subject's attention level to its maximum when a mortal threat is perceived in his environment, it becomes possible to associate the complete pro- 
gressively varying range of intensity levels of the amygdala responses to stimuli with the whole gamut of the progressively varying uneasiness levels between this extreme level and the lowest possible level likely to trigger an amygdala response.

Extensive testing involving the interpretation of electroencephalograms (EEG) revealed that what generates a feeling of uneasiness likely to trigger a response from the amygdala, is any type of unexpected or novel stimulus, which elicits a cortical event-related electrical potential variation (ERP) resulting from a pattern of limbic activity different from that produced by familiar or predictable stimuli (Peterson, 1999: p. 50).

It was observed that the polarity of the ERP waveform during the first 500 milliseconds after all unexpected or novel stimuli always shifts from negative to positive according to the same predictable inversion sequence, switching from a $200 \mathrm{~ms}$ initially negative phase to a positive $300 \mathrm{~ms}$ phase (often referred to as the N2/P3 sequence). It was also observed that the waveform during both the N2 and the P3 sequences vary with the affective significance and magnitude of the stimulus, and can even appear when an expected stimulus is not provided. As quoted by Jordan Peterson (Peterson, 1999: p. 51), neurophysiologist Eric Halgren states that:

"One may summarize the cognitive conditions that evoke the N2/P3 as being the presentation of stimuli that are novel or that are signals for behavioural tasks, and thus need to be attended to, and processed" (Halgren, 1999: p. 205).

The overwhelming evidence is now that the amygdala and the hippocampus are at the origin of the N2/P3 waveform. The most interesting discovery however, in context of the present paper, relates to verbal stimuli that are known to originate from the neocortex, and for which the limbic system rather generates a $\mathrm{N} 2 / \mathrm{N} 4 / \mathrm{P} 3$ waveform. It was observed in a conclusive manner that a negative ERP signal of $400 \mathrm{~ms}$ (N4) becomes interleaved between the P2 and P3 sequences whenever participating volunteers were exposed to verbal stimuli, whether written, spoken or communicated by means of the sign language, and that the N4 signal increases in magnitude as a function of the difficulty of integration of their meaning in the context in which they are formulated.

This means that contrary to a sensory stimulus coming from his environment triggering a P2 signal that raises and concentrates the subject's attention towards a search for more sensory information originating from his external environment, which does not require any interaction with the neocortex and is naturally followed by a P3 signal corresponding to a reaction being activated, a verbal stimulus originating from the neocortex triggers a P2 signal, that raises and concentrates the subject's attention in search of more verbal information originating from the "interior environment" of his thoughts whose origin is the neocortex, which naturally triggers a N4 signal corresponding to this search, before the P3 signal corresponding to a reaction being activated.

Moreover, the N4 signal has been identified as mainly originating from the 
left temporal lobe (Van Petten \& Luka, 2006), where the verbal areas of the neocortex are located (Figure 1), and also involves to a lesser extent the mirror areas of the right temporal lobes where the non-verbal sensory synaptic imprints are stored, and the areas adjacent to the verbal areas where the synaptic imprints of the abstract concepts are stored, and to which the words can also refer.

Figure 1 localizes in a general manner the verbal areas in the left hemisphere of the neocortex (the right hemisphere in about $5 \%$ of human beings) where the synaptic imprints corresponding to the recognized words are stored. The engrams corresponding to the motions that must be executed by the phonation organs to pronounce each word are located in the Broca area which is located just behind the left prefrontal lobe (Figure $1 \mathrm{~A}$ ). The center of the writing praxis is located a little higher (Figure 1 B), within Brodmann area 4. Finally, the area where all verbal synaptic imprints interconnect areas $1 \mathrm{~A}$ and $1 \mathrm{~B}$ with the whole collection of nonverbal synaptic imprints originating from sensory perceptions, and other nonverbal symbolic imprints that words identify and generalize is the Wernicke area (Figure $1 \mathrm{C}$, Figure $1 \mathrm{D}$, Figure $1 \mathrm{E}$ ), corresponding to Brodmann areas 39 and 40, as well as parts of areas 21, 22 and 37.

Chauchard subdivided the Wernicke area into three sub-areas (Chauchard, 1960b): 1) The sensory center of language (Figure $1 \mathrm{C}$ ), that is, Brodmann area 40, 2) The reading center (Figure $1 \mathrm{D}$, that is, Brodmann area 39, and 3) The words auditory center (Figure $1 \mathrm{E}$, comprised of parts of Brodmann areas 21, 22 and 37. The verbal areas are also summarily described in reference (Shepherd, 1994).

It seems clearly established now that a N4 signal is recorded specifically when semantic processing is required in relation to the context in which the words are used. This means that a N2/N4/P3 sequence corresponds to an effort made by the subject to more clearly understand the meaning of the sequence of words that elicited this N2/N4/P3 response; which confirms that a 3 steps physiological signal involving in sequence the amygdala, the verbal areas of the neocortex and finally the hippocampus, is required when some aspect of the verbal thought expressed by this sentence is felt to be in any sort of conflict with the set of other thoughts previously integrated on this issue, whether this sentence was heard or read originating from an external source, or simply internally formulated as the subject was mulling over this issue.

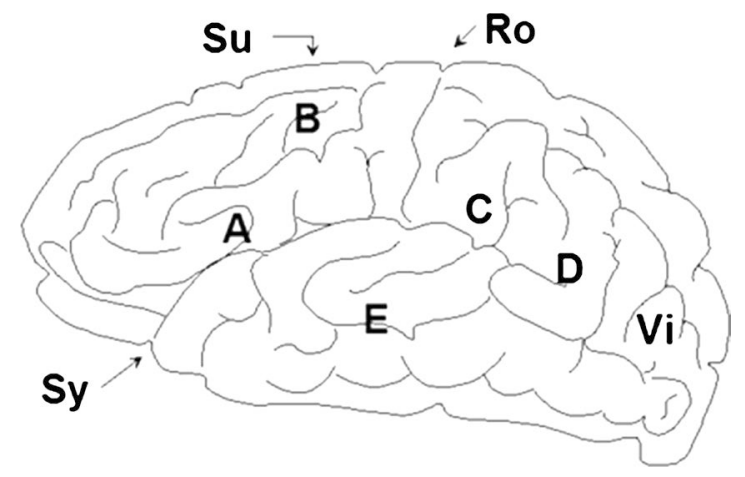

Figure 1. The verbal areas in the neocortex. 


\section{The Amygdala}

As mentioned previously, one of the primary functions of the amygdala has been identified as generating and setting of the intensity of the feeling of fear, that, when reaching a level corresponding to a feeling of mortal danger, whether real or imagined, will trigger the instinctive genetically programmed fight-or-flight reaction meant to save the life of the subject. Summarizing, this type of instinctive behavior is related to amygdala/hippocampus N2/P3 activation sequences in humans. Besides generating such feelings related to sensory perceptions, the amygdala has also been found to generate various levels of uneasy feelings related to verbal stimuli, associated this time to $\mathrm{N} 2 / \mathrm{N} 4 / \mathrm{P} 3$ activation sequences, related to neocortex verbal areas activities.

This is what establishes the feeling of fear as being a feeling of extreme uneasiness that would establish this level of intensity as the most extreme of a decreasing uneasiness intensities scale at the lower end of which the intensity levels of unease elicited by verbal stimuli can range from no unease at all when the meaning of verbal stimuli is familiar or of no interest to the individual, to any level of heightened unease that verbal stimuli can trigger, each of which thus initiating a $\mathrm{N} 2 / \mathrm{N} 4 / \mathrm{P} 3$ response.

It is well established that a feeling of intense fear, that is of extreme uneasiness, instantly triggers a release of adrenaline in the bloodstream that increases the state of active awareness and all sensory perception to heightened intensity, often accompanied by a state of rage, in cases when fight is the instinctively chosen reaction, or by a state of depression when flight is the instinctively chosen reaction. Being instinctive, this immediate fight-or-flight response occurs before the individual can even consciously decide which of the two options is the most appropriate, an instinctive choice that he can of course change after he has had some time to assess the situation.

It is interesting incidentally to note at this point that the maintained post traumatic hypervigilance of soldiers returning home or of people who experienced or witnessed life threatening situations, in fact all states of hypervigilance, seem to be related to a still not completely understood physiological condition that impedes the relaxation of these amygdala triggered physiological states even after it is conceptually understood by the subject that the physical danger at the origin of such a state is no longer threatening.

But contrary to extreme unease levels related to perceived imminent life threatening danger, the lower intensity levels of unease induced by verbal stimuli do not trigger such uncontrolled reactions, but rather simply draws the attention of the subject to the meaning of the words that induced this uneasiness feeling, awaking feelings of curiosity that raise the level of interest instead of the level of fear.

It appears that physiologically, the amygdala then activates a process of excitation in the verbal areas of the synaptic network of imprints related to the stimulating word sequence, resulting in a relative inhibition of the synaptic connec- 
tions to all other verbal synaptic imprints (Chauchard, 1963b: p. 82), which polarizes and orients all neural activity towards a recollection of the memories related to the stimulating words sequence.

Contrary to sleep that can be considered as an inhibition wave submerging the whole neocortex, attention, that is, active awareness, can be seen as a narrowing of the subject's awareness to the arborescence of synaptic imprints related to the specific sequence of words that triggered the feeling of unease, by strengthening the synaptic links only between the imprints belonging to the arborescence, which inhibits by repercussion the synaptic connection between this arborescence and all other imprints in the neocortex (Chauchard, 1963b).

Conclusive research has also shown that although the amygdala is rather heavily interconnected with most brain structures, it is most strongly interconnected with the prefrontal cortex and the temporal structures related to language recognition, which comes in support of the conclusion that the N2 excitation phase triggered by the amygdala uses this heavily interconnected network to causes the activated arborescence to be linked to the entry layer of the prefrontal cortex, which is known to be involved in decision making (Bickart, Dickerson, \& Feldman Barret, 2014), as a single set to be simultaneously processed, in conformity with the initial phase of simultaneous processing of sets of elements by multilayer neural networks identified by Donald Hebb (Hebb, 1949).

In conclusion, it could be considered that the amygdala is the "alarm system" of the human body, that triggers into action each time that an unexpected situation is signalled via sensory perceptions stimuli coming from the external environment, or via verbal stimuli initiated by internal cogitation or coming from outside sources such as verbally formulated information being read or heard, of even felt such as any Braille text in process of being tactilely read.

\section{The Attention Mechanism}

As mentioned previously, the attention mechanism is the physiological process triggered by the amygdala that increases the level of active awareness about some unexpected sensory perception or verbal stimulus whose meaning is unexpected, which elicits a feeling of unease generated by the limbic system and causes the amygdala to activate. This increased level of active awareness causes the subject to remain concentrated on identifying the cause of this discomfort in view of resolving it, compared to the lower level of awareness that characterizes our default haphazard roaming less intently in the network of our memories as when we daydream.

The attention process was observed as acting somewhat like visual perception. Similarly to eyesight, that provides clear visual perception in a restricted area in the field of vision, that progressively goes out of focus as the distance increases from this clear visual area, our heightened level of attentive awareness encompasses only the restricted field of the arborescence that was activated, while the level of awareness of other memories progressively fades to half awareness and 
finally to complete unawareness of any other issue.

"Attention is a unified orientation process of behavior, it involves a redirection of the processes of static and dynamic activity in a given direction combined with a discontinuation of activity in all other possible directions, that is, an inhibition of any form of behavior not in line with the dominant orientation ( $H$. Piéron). Inversely, the other neurons, the other types of referral, will be inhibited, whether they simply remain at rest with respect to the newly excited orientation or are voluntarily inhibited" (Chauchard, 1963b: p. 82).

According to Chauchard and his colleagues (Chauchard, 1963b), at the physiological level, attention is the process that causes us to become intensely interested about the various aspects of an action, object, concept, or event, verbal or non-verbal, that unexpectedly makes us uncomfortable when thinking about it.

"While sleep could be considered as a wave of inhibition submerging the neocortex, attention, thus awareness would be a wave of excitation" (Chauchard, 1963b: p. 83).

So, sleep can then be defined as wave of inhibition submerging the neocortex, leading to a loss of active awareness of all of our memories, accompanied by loss of active awareness of our sensory perceptions; wakefulness as a wave of excitement that pervades the entire neocortex leading to a state of active awareness of all of our memories and sensory perceptions; and attention as localized wave of overexcitement leading to a state of heightened active awareness of part of our memories, accompanied of loss of active awareness of our other memories, and often by momentary partial or even complete loss of awareness of our sensory perceptions, amounting to concentrating all of our active awareness to a single issue at the exclusion of all other considerations.

\section{The Verbal Areas of the Neocortex}

After the over activation of the arborescence of synaptic imprints leading to the memories related to the words that induced the verbal stimulus, the question comes to mind as to what sort of processing will occur functionally and physiologically in the neocortex during the $\mathrm{N} 4$ phase of the amygdala/neocortex/hippocampus sequence before the hippocampus enters in action. The resolution of the discomfort that triggered the verbal stimulus involves by definition that a process of "learning" needs to be involved that will generally result in a change in understanding of the phrase as formulated, that could take the form of the acquisition of a new conclusion, clearer recognition of a situation, refinement of a gesture, etc.

Strangely, the correlation that can be made between the discovery by Donald Hebb in the 1940's (Hebb, 1949) that the neocortex is a 6-layeur neural network and the prior discovery by Pavlov in the 1930's that articulated language sustains conceptual thinking from within this network (Pickenhain, 1998), seems to have raised little interest in Pavlov's own field, that is, that of neurophysiology. Hebb's discoveries rather served as a foundation for intensive research in the 
domain of artificial intelligence (AI), recently culminating in the development of the advanced technique of deep learning (Goodfellow, Bengio, \& Courville, 2016), particularly effective in the development in exponential growth of all sorts of increasingly efficient AI applications.

His discoveries about the neocortex are again referred to in a series of recent articles (Shepherd, 1994; Michaud, 2017b; Anderson, 1995), of which the present paper is the fourth and last component, in relation with the development of the understanding ability in human beings that was explored by Chauchard and his colleagues in the 1940's and 50's (Chauchard, 1963a; Chauchard, 1963b; Chauchard, 1960b; Chauchard, 1970a; Chauchard, 1944; Chauchard, 1960b; Chauchard, 1970b). This series of articles is meant to draw renewed attention to the fundamental role played by these properties of the neocortex in the emergence and development of conceptual thinking in human beings.

The four correlation properties inherent by structure of all multilayer neural networks, that is, the structurally built-in automatically applied discrimination criteria of simultaneity, successivity, similarity and dissimilarity (Blayo \& Verleysen, 1996: p. 23), are applied to any set of elements provided at the entry layer of the network, as their signals are compared while they travel within the thickness of the 4 intercalary layers of the neocortex, leading to coherences made available at the $6^{\text {th }}$ layer, that is, the output layer; coherences established by the automatic correlation process, and which is the only information that we can become actively aware of from our sensory perceptions (Michaud, 2017b).

On the other hand, four specific activities have been identified as sustaining the learning process, which is the process of acquisition of knowledge about the environment, that is, association, classification, discrimination and assessment. The first three, that is, association, classification and discrimination are guided by the generalization ability emerging from the use of articulated languages. Unsurprisingly, these three activities are carried out as a function of the same four correlation criteria that are inherent to the functioning of the neocortex, that is, simultaneity, successively, similarity and dissimilarity. The fourth activity, that is assessment, will be analyzed in the next section, but let us first pay attention to the four correlation criteria.

The criterion of simultaneity establishes the spatial relation between an element and other elements being correlated. This criterion, besides intuitively causing in infants the emergence of the spatial sense by the observation that different objects (dissimilarity) in their environment are simultaneously present (similarity) at different locations (dissimilarity); it is this criterion that causes the emergence of the number sense previously mentioned, when the child begins to understand that different objects (dissimilarity) located at different locations (dissimilarity) in their environment have the same name (similarity), which by structure mandatorily involves the use of verbal stimuli.

"To each image is substituted through education its verbal denomination. Since they name everything, instead of associating images, human beings can directly associate the corresponding names, a system more adequate to fully ex- 
tend the abstraction capabilities of the human brain" (Chauchard, 1960b: p. 122).

Since that as young as 6 months of age, children already begin to think with the names that adults about them give to objects that they observe in their environment (Bergelson \& Swingley, 2012), it becomes natural for them to already elaborate and stabilize the first level non-verbal abstract mathematical concept of multiplicity of different objects (dissimilarity) about which he now thinks of by means of a single word (similarity), whence the emergence of the number sense.

As already mentioned, from the moment that a child understands that the single word "chair", for example, identifies many different objects in his environment, the criteria of similarity and dissimilarity that he can apply to each of their individual characteristics (color, shape, etc.), will allow him to identify them individually, but the criterion of similarity repeatedly applied to the unique name itself that comes to his mind each time that he observes these objects after having associated this unique name to each of them, can only repeatedly strengthen his understanding that more than one object corresponds to this unique identifying criterion.

The criterion of successivity establishes the temporal relation between an element and other elements being correlated. Just like the simultaneity criterion intuitively causes the emergence of the spatial sense in infants without any need for verbal stimuli, the successivity criterion intuitively causes the emergence of the time sense without any need for verbal stimuli either (Droit-Volet \& Coull, 2015). The observation that objects can be only intermittently or cyclically present, intuitively causes the emergence of the time sense.

Indeed, it was observed that even shortly after birth, infants can perceive the length of a time interval between two occurrences of an event regularly repeated (Blackbill \& Fitzgerald, 1972). Moreover, a study published in 2010 by Elizabeth Brannon et al. confirms that from the age of 6 month, this intuitive earlier development of the time sense starts developing at the same rate as the number sense that first appears only from this age onwards (Brannon, Suanda, \& Libertus, 2010). This means that becoming aware of the abstract concept of the existence of processes that by very definition imply a change of state in the environment, which is thus directly linked to the time sense, is also linked to verbal stimuli.

On their part, the similarity and contrariness criteria are the very foundation of the generalization ability provided by mastery of language, as summarily demonstrated while analyzing how the criteria of simultaneity and successivity are used during the automatic correlation processes used by the association, classification and discrimination activities of the neocortex.

Indeed, they are the binary opposite conditions $(1,0)$ that allow defining how the first 2 criteria of simultaneity and successivity apply to the various characteristics of objects and processes in our environment. They allow clear discrimination between the fundamental static versus dynamic characteristics of these $o b$ - 
jects and of the processes that can now be more clearly described with the more advanced non-verbal geometric/mathematic language that has now been developed from their verbal descriptions. See Section "The mathematical thinking mode" for deeper analysis.

The metaphorically "perpendicular" criteria of simultaneity and successivity can also be seen as binary opposites $(1,0)$ that when correlated with the two other opposite criteria of similarity and contrariness $(1,0)$, could even be seen as a 4 -states code that might well possibly be the very coding method used by the neocortex to encode the synaptic imprints of each element stored in memory, whether verbal or non-verbal, in a manner reminiscent of the genetic code; possibly in fact, an arborescent transposition of the sequential coding method of the genetic code to store information.

These four selection criteria are apparently all that is required for a human being to successfully process all of his sensory perception as he converts them into his personal subjective spatiotemporal model of the objective physical reality that he needs to adapt to in order to survive.

From the moment that a child begins to master his mother tongue (more than one language for many children), any unease with the meaning of a verbal stimulus triggers a low level emotional urge to resolve the conflict that he perceives between a new word, or an already known word and information concerning any object, event, chain of events, abstract concept or emotion that he previously integrated and harmonized, which progressively enriches his subjective understanding of physical reality towards a level minimally sufficient for successful interaction.

\section{The Hippocampus}

Let us now examine the fourth activity required to complete the process of learning, that is, the assessment activity (Blayo \& Verleysen, 1996: p. 23), that comes into play only after the resulting coherences have been established in the prefrontal cortex. Once one or more possible "solutions" to the discomfort that triggered the amygdala into initiating a N2/N4/P3 sequence, the hippocampus automatically takes over to automatically select which resulting coherence is the most emotionally satisfying.

Although the hippocampus selection mechanics is fully automatic by default only as a function of the emotional response elicited by each of the resulting coherences provided by the prefrontal cortex, since the individual is in the process of paying active attention to the process, he can thus of course consciously assess the set of resulting coherences and can of course force a logical preference of final solution, even against the automatic "emotionally intuitive" selection suggested by his hippocampus.

The subject can even reject all coherences obtained in first instance and force complete reassessment by increasing or reducing voluntarily the set of elements in the set to be considered, which is precisely what the process of "cogitation" 
involves, to finally let the hippocampus' "emotionally intuitive" choice operate only when the subject is conceptually satisfied with a newly obtained coherence, that he now prefers even emotionally.

Besides automatically selecting the emotionally most satisfying action to be taken from a choice of options offered by the prefrontal area, unless this choice is voluntarily countered by the subject, another major function of the hippocampus was clearly identified and confirmed, in context, following the historic case of a patient referred to in the scientific literature as H.M.; who had both left and right hippocampi removed in an attempt to cure his epilepsy.

It was observed afterwards that H.M. had become unable to accumulate any new memory, either long-term or short term (Anderson, 1995: p. 153). Even if he behaved normally in all respects and was able to hold long continuous intelligent conversations with a stranger, after that person left the room for only a few minutes, H.M. had become unable to remember having had this conversation or of having even met the person with whom he had discussed.

This confirmed that while the hippocampus is in activity during the P3 phase, besides selecting and activating the most emotionally satisfying action to be taken, it also strengthens and permanently stabilizes in the neocortex the complete synaptic network of the memory corresponding to this action as a function of the intensity of the emotion that it elicits, which may or may not involve physical action on the part of the subject, such as in the case of reactions to danger or in the case of conversations with other individuals, for both short and long term stabilization of the memory of the action concerned.

This means that the hippocampus is the default decision-maker as to the action to be taken among the choices proposed following the state of alert triggered by the amygdala, signaling either that an action must be taken with respect to sensory perceptions, or that a choice must be made with respect to the meaning of a verbal stimulus.

In case of extreme physical danger alerts signalled by the amygdala, presumably from sensory perceptions, the genetically programmed instinctive protective reactions of either fight or flight are the only options offered as possible choices by the hippocampus as the most likely to save the life of the subject, out of reach of the immediate voluntary control of the subject. All other less intense alert levels of the amygdala however obviously leave some time for the subject to assess situations.

This choice will usually be in full agreement with the default emotional preference of the subject, guided by the emotional signals originating in his limbic system, as to which solution among those offered feels the most emotionally rewarding, satisfying, etc. or alternately, the less displeasing, less painful, etc.; but since the subject can also evaluate situations according to other information previously memorized, he can also voluntarily override this default hippocampus emotional choice, by introducing a parameter that logically, that is non-emotionally, causes a different option to become the most rewarding, satisfying, etc., or alternately, the less displeasing, less painful, etc., in this specific case. 
It is even possible that no action may be taken about any amygdala alert short of maximum physical danger signal, due to a momentary loss of interest in a particular stimulus caused by to a different stimulus having triggered the amygdala alert about a different issue.

\section{The Origin and Function of Emotions}

Two separate action sequences of primordial importance are genetically programmed in the neural sub-networks of the human limbic system, which is known to have evolved from more primitive cerebral structures present in more primitive mammals and vertebrates from which our species genetically evolved; the first controlling the instinctive fight-or-flight behavior meant to insure the physical survival of the individual, and the other controlling the instinctive reproductive behavior meant to insure the survival of the entire species.

When imminent physical danger is perceived, an intense feeling of fear is induced by the limbic system that triggers immediate instinctive reactions of protection in individuals, ranging from remaining completely immobile, to fleeing, to violent fight reactions, whichever behavior instinctively momentarily feels the most likely to insure survival.

Impressions of danger of whatever nature not immediately life threatening will induce a whole range of feelings of less intense fear, associated to various degrees of displeasure or discomfort that will determine the behavior of individuals in a less immediate and instinctive manner. For example, hatred, anger, anguish, annoyance, embarrassment, despair, frustration, panic, resentment, etc.; in summary, any negative emotion that can be related to feelings by the subject that his well being or survival is at stake, whether the cause is imagined or real.

Imagined or real refers to the fact that since all sensory perceptions are filtered by the limbic system before the individual can become aware of them, some perceptions may be wrongly interpreted as being menacing due to a momentary emotional state induced by previous unrelated perceptions, which may induce uncalled for defense reactions.

On the other hand, the instinctive reproductive behavior of individuals, genetically programmed to insure the survival of the species, can be related to various degrees of pleasure that will determine the behavior of individuals in view of insuring the survival of the species. For example, emotions such as acceptance, affection, desire, empathy, joy, love, etc.; in short, all emotions that can be related to the process of selecting, attracting, keeping, and protecting a mate of the opposite sex in view of begetting descendents can be associated to various degrees of pleasure, that determine the behavior of individuals.

The so "mysterious" emotion of love, for example, can easily be traced as stemming from an instinctive need to "protect from harm" a mate and the children begotten with this mate; thus extending the reach of ego-centered protective instinctive fight-or-flight behavior of individuals to enclosing the mem- 
bers of the immediate family circle, a behavior that may eventually extend to widely larger groups (Michaud, 1997).

By opposition, the origin of feelings of hate can be traced by a similar correlation to the irrepressible urge, begotten by fear, to protect them from anything that would be interpreted, rationally or not, as threatening their lives or well being as if they were one's own.

In summary, the highest intensity level of these apparently opposite emotions of love and hate, stemming from these reflexes genetically programmed to insure individual and species survival, could be seen as the two limits of the whole spectrum of all possible intensity levels of opposite emotions that could be elicited by real or imagined impressions of danger to survival or well being of self, extended to the family circle if the case may be, on one hand, and by success in insuring the survival of the individual and the family circle and fulfilling the urge to reproduce and thus insuring the survival of the species, on the other hand.

Indeed, due to the extreme complexity of the whole gamut of emotions that human beings can feel in relation to their sensory perceptions, any of these feelings can end up being applied to all sorts of situations and objects, that may not even be remotely related to survival of the individual or of his family, and that were initially established by Nature as instinctive behavior to insure this survival, and thus color all sensory perceptions of each individual in unexpected ways.

Given that the hippocampus is genetically programmed to choose by default either the most emotionally satisfying action, or failing this possibility, the less displeasing action, even an action logically chosen by an individual in contradiction with a possible choice of first selection, becomes de facto the most emotionally satisfying for the hippocampus at that moment, because this choice is now the one that consciously most satisfies the individual.

Incidentally, it is the absence in artificial neural networks of such an uncircumventable selection mechanism of the most pleasing or alternately the less displeasing option that characterizes the neural networks of living beings, that explains why artificial neural networks are unable to draw any conclusion that they have not been trained to draw, despite the development of leading edge training techniques such as deep learning (Goodfellow, Bengio, \& Courville, 2016).

In the domain of instinctive reproductive behavior, perceptions can also turn out to reflect reality or not, that is, being real or imagined, and involve more complex situations than the instinctive fight or flight behavior meant to insure individual self-preservation, because instinctive reproductive behavior involves by definition the coordinated emotional behavior of two individuals, and the behavior of a potential mate can easily be wrongly interpreted as being welcoming, or unwelcoming.

\section{The Function of Articulated Language}

Up to now, we have been specifically analyzing the process by which one neural 
N2/N4/P3 amygdala/neocortex/hippocampus activation sequence allows drawing and storing in memory one conclusion at a time, that is, one conclusion each time some unease regarding some verbal stimulus activates the amygdala.

This could easily leave the impression that each conclusion that we draw is stored within memory as a separate item, since it can be clearly established that the whole collection of praxis and various other synaptic imprints, aka engrams, pertaining to any given word weave a clearly separate and identifiable synaptic network for each of them, a web that spreads "horizontally", so to speak, within the thin neocortex, between the various verbal and nonverbal areas of the verbal hemisphere, interconnecting these words with the non-verbal "images" corresponding to our sensory perceptions mostly located within the mirror Wernicke area of the other cerebral hemisphere.

Of course, each word learned, and each verbal conclusion drawn, also defines at the functional level a very precise subnet of synaptic interconnections that correlates all of the elements that lead to that word or that verbal conclusion, and which can thus be collectively brought to the subject's attention when he thinks of this word or correlated verbal conclusion (Michaud, 2003). Each word, correlation or conclusion that a subject uses to think with is thus functionally synaptically also connected "vertically", so to speak, with the complete subset of related verbal or non-verbal elements that were correlated in the past to establish its meaning.

It is indeed this particular "metaphorically vertical" biological interconnected synaptic structure, associative by inclusion, that relates any conclusion drawn to the entire subset of grounding elements that allows so easy confirmation of this conclusion, by allowing easy back tracing of all supporting arguments, each of which also connected to its own subset of supporting grounding arguments allowing verifying its validity, and so on, until the validity of the whole structure has been established. Any element found invalid in the extended substructure of any conclusion obviously will jeopardize the validity of this conclusion and of any other conclusions of which this particular conclusion would be a grounding element.

Presently, the whole collection of our conclusions are associated in this manner into an incredible hierarchical structure, sort of a metaphorical virtual pyramid, within which they associate uniquely by inclusion; an invisible structure that constitutes in fact our personal subjective neurolinguistic model of reality, that takes shape within the neocortex of each person from birth onwards, according to the manner in which the person progressively associates and constantly refines his verbal descriptions of his environment.

Such a structure is apparently the only way that information can be verbally interrelated in multilayer neural networks, which is a conclusion that was also reached by Jeff Hawkins and Sandra Blakeslee in a work published in 2004 (Hawkins \& Blakeslee, 2004). The number of levels that such a verbal indexation structure can have is unlimited for all practical purposes, due to the fact that the 
neurons of the neocortex can establish any number of reverse output axon synaptic connections with the dendritic inputs of neurons of preceding layers in the 6-layer structure.

In waking state, our active awareness appears to be constantly circulating in this extraordinary network among the interconnected engrams within which travel is made easy by the frequent re-strengthening of the synaptic pathways that associate them (Hebb, 1949), each engram corresponding to a word related to the arborescence of the non-verbal imprints (sub-engrams) whose coherence was previously associated to this word; jumping at will from one engram to another through the synaptic pathways provided by any characteristic that happens to be common to more than one engram (Michaud, 2017b).

When our attention is attracted to an idea by the amygdala activating upon any level of unease with respect to its verbal stimulus, typically a sentence, the whole complement of the separate arborescences of each word involved will be simultaneously activated:

"The other types of branching are then inhibited, either because they remain at rest with respect to the new excitation, or that they are voluntarily inhibited" (Chauchard, 1963b: p. 82).

"The neurological aspects of the thinking process are the variable structuring established in the network through the interplay of the states of excitation and inhibition. They are neuronal sets that are subjected to waves of excitation and inhibition, either localized points in the network, or interconnected groups in the whole cortex" (Chauchard, 1960b: p. 63).

So when an unresolved aspect of an idea attracts sufficiently our attention to induce us to question the subject, each question activates other promising arborescences and/or deactivates conflicting arborescences of specific elements of the set, resulting in a new configuration that will ideally give the subject a better understanding of the vague aspect that had attracted his attention, which depends of course on whether or not coherent answers were identified by the questioning (Michaud, 2017b; Michaud, 2003).

It seems that each unresolved question then triggers the establishment of a new separate correlation process that will be the seed of a new arborescence to be completed; which is demonstrated by the fact that these unresolved questions continue re-attracting our attention each time one of the elements already included in this partial arborescence is activated even indirectly while cogitating or discussing about unrelated issues.

The ease of change in the orientation of the thread of coherent thinking is an inherent characteristic of the hierarchical structure associative by inclusion that the use of articulated language establishes between the various aspects of the sequences of events that are recorded in our memory as a result of our sensory perceptions, and also with the abstracts concepts that our conclusions define to generalize them. It is this versatility that explains the incommensurable power of the human comprehension ability.

The richness of this structure, in conjunction with the rigor of the logical ap- 
proach employed, determines the degree of ease with which each individual will be able to become aware of the coherences that the neural networks of the neocortex automatically detect and correlate independently of our will in any set of elements that clear identification of whose reference frame allows to circumscribe, as extensive as it may be.

A correlation process thus consists in a cascade of successive associations that will gradually "bring to the attention" of the subject a set of pertinent elements (of course, only those that the person has become aware of and considers valid), and when a "satisfying" coherence has been perceived by the subject, which manifests itself by an activation of the $\mathrm{P} 3$ phase of the processing sequence, the set of synaptic interconnections that associates these elements will naturally constitute a new arborescence, that is, a new hierarchical substructure within which each of the synaptic links leading to one of the elements of the set will be included and permanently strengthened following the over-activation by the hippocampus of this "satisfying" coherence, to become a separate newly integrated engram.

Afterwards, the previously unresolved aspect of the idea that had triggered the questioning, instead of activating the question, will directly activate the newly stabilized and re-enforced arborescence of the elements whose coherence now constitutes the answer to this question.

\section{Subjective Perception of Physical Reality}

The question that now comes to mind is how to define what really exists in our environment, that we identify as being "physical reality", or "objective reality", whose signals we each individually detect by means of our separate individual sensory perceptions and that each of our individual "attentive awareness" makes us collectively conclude are physically existing objects distributed in space and involved in processes progressing over time.

Given the physiological fact that any information that reaches our individual brains coming from our environment can only originate from excitation induced in each of our individual nerve endings, absolutely all of what our individual awareness can observe can only be the collection of all memories stored as synaptic imprints in our separate neocortices, memories that we individually abstracted from this information personally perceived.

This information is constantly being collected by clusters of nerve endings genetically cabled to the entry layer of specific parts of the brain. After having been automatically correlated and synthesized as feelings of smell, hearing, taste and touch by dedicated multilayer neural networks, these feelings are filtered by the limbic system before being converted to "mental images" as they are correlated with our simultaneous visual perceptions, and made available for our active awareness to observes and react to.

The case of our eyesight is unique and different from the other senses in that the entry neuron layer of the visual cortex is located directly within the eyes re- 
tinas and is directly excited by light entering the eyes, without going through the limbic filter.

The rather complex processing to which is submitted each of the countless millions of individual submicroscopic nerve impulses on its way to become part of the "macroscopic image" that our awareness perceives in our environment reveals that despite our deep conviction that we are observing our environment "live", so to speak, this is far from the case. At best, our active awareness is observing freshly stored memories of events and objects as they were a few milliseconds in the past, that is, the time it took for the resulting images to be stored in the neocortex for our active awareness to become able to perceive them.

For example, let's observe that at this very moment, each word of the sentence that the reader of these lines is in process of reading are being recorded one by one as his reading progresses, activating as he goes the arborescences that he associates to each word, and the complete sentence is already "a stored past memory" when he reaches the last word and is already cogitating about its meaning.

Regarding this particular sentence, it must be observed that it is not the arborescences that the writer had in mind for each word that comes to the mind of each reader, but those that each reader had constructed himself over the course of time and that he associates to these words. They may well coincide when simple ideas such as this one are conveyed, but when complex ideas are exchanged, the situation is quite different as exemplified by the experiment previously mentioned carried out by Amalric and Dehaene (Amalric \& Dehaene, 2016).

The conclusion is that words do not carry the meaning a speaker or writer assigns to them, but the meaning that the listener or reader does, which can lead to important misunderstandings of complex issues if the specific meaning of each word is not clarified (Michaud, 2017b), since the extent and orientation of the specialized knowledge base in any given direction of each person can vary widely. This is what makes the model of reality of each person a subjective construct, related only to what each person has abstracted from the sum of his own perceptions, emotions and interpretations.

\section{Objective Perception of Reality at the Personal Level}

The possible emotional bias induced by the limbic filter combined with the fact that our sensory perceptions may not have let us pick up all the information required for objective perception reveals that there is no guarantee that each person observing the same event will perceive and interpret it in the same manner and with the same level of clarity. Clearly, the more information is gathered about any situation, the more probable clear understanding becomes likely. On the other hand, it is consistently observed that unless the possibility of an incorrect perception is specifically brought to their attention, witnesses of specific events tend to be certain that they have correctly perceived these events.

A property specific to the manner in which memories are stored as arrays of strengthened synaptic links within the neocortex must be brought to attention at 
this point. The strength of synaptic links within living neural networks is known to naturally diminish over time if they are not regularly re-visited, which tends to cause memories to slowly fade from easy recall as times passes, and to eventually be forgotten if not re-activated for very long periods of time.

This property further complicates the issue of certainty about memories, given the automatic correlation mechanics of multilayer neural networks discovered by Hebb (Hebb, 1949). Due to this natural process, when some memorized aspects of past observations become too faintly connected to be easily reactivated, the network automatically tends to reestablish a coherence to harmoniously reconnect what can easily be re-activated of these memories, subconsciously constructing "replacement segments" to logically harmonize the segments that can be remembered of past events, which is a phenomenon that has regularly been observed in courts of justice regarding the progressive evolution over time of the versions of witnesses that cannot be suspected of acting in bad faith (Lacy \& Stark, 2013).

Paradoxically, this is precisely what gives us the key to determine if any given perception is conforming to objective reality. Taking on the habit of never being "totally certain" of having correctly remembered, correctly perceived or correctly understood any given situation or event keeps people alert to any possible additional information that might cause them to reach a possibly more accurate conclusion about this situation or event, or to rectify an altered recollection, by verifying with other people who may have witnessed the same situation or event. A detailed description of how this requestioning process can be used is given in separate references (Michaud, 2016a; Michaud, 2017b).

Clear evidence also strongly suggests that the greater the mastery level of his mother tongue by a person, the clearer and the more detailed his analysis of any situation or event is likely to be (Michaud, 2016a; Michaud, 2016b; Michaud, 2017a). Moreover, it was conclusively demonstrated that the earlier in life this mastery is acquired, the more beneficial this tool will be for the rest of a person's life (Chauchard, 1960b; Dodson, 1971).

Finally, at the physiological level, a major discovery by Paul Flechsig in 1920 that complete myelination of the verbal areas of the human brain is genetically delayed after birth until the age of about 7 years (Flechsig, 1920), may explain why children in multiethnic families easily learn more than one mother tongue in youth to similar mastery levels, and also why children who have not mastered writing of their mother tongue to autonomy by this age experiment increased levels of difficulty in completing later this training as well as other verbal trainings.

This remains to be confirmed of course, but it appears quite possible that construction of synaptic connections in these areas is made easy by not being hindered during the first seven years of life by the physical presence of the myelin sheaths about the neuron axons. If eventually confirmed, this would reveal that all verbal skills need to be mastered to autonomous control before the end of this period for children to benefit from their optimal verbal potential, which seems to 
be borne out by the fact that all children who benefit from such early training all perform exceptionally well in school, and generally also later in life (Chauchard, 1960b; Dodson, 1971; Piaget, 1974; Piaget, 2001).

\section{Objective Perception of Reality at the Collective Level}

At the collective level, it is the coinciding conclusions drawn by many that constitute the sum of mankind's collective knowledge, now regrouped into the various scientific domains.

This sum of conclusions was of course rather limited at the beginning of humanity and has increased over the course of time up to the larger set of information and conclusions that we are currently using collectively. Some of these conclusions have been proven out of any doubt and are recognized by all while others are still uncertain or may even be objectively outright false without our having been able to determine yet. Each of us ends up making his own subjective opinion about each of these conclusions. As more and more information is gathered about our environment, uncertain conclusions that are possibly objectively right end up being collectively confirmed, and those that turn out to be proven objectively false end up being collectively rejected.

Since the totality of the information that we can individually abstract from our environment from our sensory perceptions can only be abstracted by structure from subjective past memories, that these memories are established through the filter of our emotional states, that they are subject to fade from memory over time, to be unexpectedly interspersed by arbitrary replacement segments, this sum of personally accumulated information may not have let us become aware of all of the objectively important characteristics of the observed objects and processes, so the following question comes to mind.

How can objective knowledge be ascertained at the collective level about our environment, since there is no guarantee that each individual will have perceived all objectively important characteristics of existing objects and processes and would have interpreted any given event in the same manner and with the same level of clarity?

The answer to this question seems to reside in the coherence that we perceive in the fact that we collectively observe that all human beings are simultaneously observing the same collection of objects and processes in their environment everywhere where they also are in a position to simultaneously observe each other in a confirmable way; from which observation can be concluded that there exists only one objective reality that we only need to become better acquainted with until all of its objectively important characteristics have been identified.

This means that it is possible to collectively double check ad infinitum all characteristics of any object or process to confirm their stability, until the preponderance of the evidence confirms that all of their objectively important characteristics have been accounted for and understood. The reference to verify this stability is repeatability always providing the same outcome in space as well 
as in time, regardless of the number of such tests and regardless of how many individuals independently carry out the testing.

In the very specialized domain of physics for example, the electromagnetic equations developed by Coulomb, Gauss, Ampere, Faraday, Maxwell, Biot-Savart and Lorentz, constitute a set of convergent equations developed from the repeated analysis of data obtained from such endlessly verifiable experiments always giving the same result. In addition, their conformity with objective reality is confirmed out of any possible doubt by the very fact that all of the successful modern electric and electronic technology that we all benefit from is exclusively grounded on these equations. They then constitute such a constantly verifiable invariant objective reference that we can refer to establish what remains to be understood about objective physical reality.

The fact that these equations harmoniously converge in their confirmed exact descriptions of various aspects of fundamental energy is then a convincing telltale that convergence itself is another trustworthy guide in our collective search of what remains to be understood about objective reality. This would suggest that any yet to be confirmed uncertain conclusion about our environment that would imply a contradiction with this established and confirmed set might de facto be invalid, and that contrariwise, that any new discovery or unconfirmed conclusion that appears to harmonize with this set would show promises of being valid, and would be likely to be at the origin of more beneficial applications yet. Convergence and repeatability thus seem to be stable and invariant guides that could be trusted in our search towards better understanding of physical reality. This example is drawn from the domain of physic, but of course, the same guidelines of repeatability and convergence would be applicable to all domains of science.

\section{The Generalization Ability}

Actually, we are able to think directly with the first level nonverbal "images" conjured up from our sensory perceptions, and with the also nonverbal first level idealized "abstract images" that we conceive of to think in a generalized manner about these first level perceptions conjured up from our sensory perceptions, such as the idealized idea of a "circle" for example, that we relate to all bodies that we think of as being "round", which constitutes the first system of signalization defined by Pavlov (Pickenhain, 1998).

But we can also think directly with first level "verbal descriptions" of these nonverbal images of all perceived or idealized objects and processes, or with second and further levels of verbal generalizations, that organize and regroup these individual objects and processes in various ways into sets that we can mentally cogitate and discuss about at will, which constitutes the second system of signalization defined by Pavlov (Pickenhain, 1998).

In conclusion, every human being naturally uses these two systems of signalization that have genetically been put at our permanent disposal, that is, the 
thinking mode by "images association" and the thinking mode by "words association". Both modes are so intimately interconnected in the entire neocortex, that any attention given to one of them triggers the immediate and simultaneous activation of the other. For example, it is not really possible for any of us to think of the word "round" without the nonverbal idea of "roundness" coming to mind, and the reverse is also true.

In the last section of this paper, titled "The Mathematics Thinking Mode", will be discussed the third system of signalization, thus named because it is now confirmed that individuals who benefited from deep training in mathematics develop in parallel the ability to directly think with this nonverbal symbolic thinking mode that allows further generalizing the processes observed from our sensory perception, in correlation with the idealized geometric dimensions, shapes and volumes of all sorts that have currently been generalized and collectively standardized (Amalric \& Dehaene, 2016).

A verbal generalization is a word or verbal expression that identifies a group of objects that share at least one characteristic in common. The generalization ability provided by articulated language can occur according to two different modes. The most common mode is generalization by context. It involves varying numbers of objects or processes, whether concrete or abstract, that possess characteristics in common, and that are implicitly included in the set being referred to by a word as determined by the context in which the word is used. This type of generalization thus involves by structure a single set of objects whose number of elements can vary from two items to an undefined number of elements.

The other mode is generalization by definition, or by inclusion. This mode can be defined as a definition of a specific and limited set of characteristics that are common to a limited set of objects or processes whether concrete or abstract. In other words, it describes the reference frame of this set of objects or processes.

\section{Generalization by Context}

As mentioned previously, articulated language is the means by which we name every object and process that we identify in our environment as well as in the abstract representations that we conceive of, which automatically regroups them into generalized categories according to their similarities or differences.

Let us take the example of the word "chair" already mentioned, that can be defined as any convenient piece of furniture that can be used to sit on and be comfortable while eating, working at a table, attending to some event, etc. This definition is obviously very general. It is in fact a generalization of the idea that the word "chair" conveys. The extent of the numbers of types of chairs that it actually describes, whether used in writing of verbally, depends entirely on the context of the related sentence. It might refer as well to all chairs in existence, to a special make of chairs, specifically to wooden chairs, and so on.

The limit is reached when a single object is identified by a word, that then ceases to be a generalization by context to become the identifier of a single ob- 
ject, that is, a first level identifier, that minimally possesses at least one characteristic that it doesn't share with any other object, if only its physical localization in space, and that activates to the level of active awareness the specific arborescence of the nonverbal characteristics of this unique chair, at least those that are activated in context. Indeed, uniqueness in the case of two objects in the environment identified by the same name, even if they are visually identical to the point that they cannot be distinguished from each other, necessarily possess the different characteristic of being located in two different places.

This description is given to clearly highlight the unbounded generalizing power of articulated language, since the same generalizing property can be applied to every word in any sentence that can be formulated, and whose extent of generalization depends entirely on the general context of the conversation, with final extension clarified by the sentence itself, keeping in mind that the context meant by the speaker might be different from the context understood by the listener if the speaker is not careful in insuring that this context is clearly made obvious.

It seems also that all spoken languages allow expressing any possible shade of meaning that an individual wishes to express. All languages, as a matter of fact, allow the construction of all sorts of "on the fly" locutions to express fine shades of meaning that words already defined in that language may not allow to directly express.

The ability to understand and express fine nuances of meaning on any issue obviously goes hand in hand with the level of mastery of the mother tongue. So the more extensive the mastery of a person's mother tongue, the more clearly a person will be likely to finely understand and describe any issue considered (Chauchard, 1960b; Michaud, 2017b).

Every spoken language possesses a unique structure that allows easier and clearer perception of some aspects of reality, that are often not as immediately obvious in other languages. A striking and extreme example of this situation is the well known case of inuktitut, the dominant language of North America's arctic people, in which can be found hundreds of different words to precisely describe the various states of water, ice and snow.

Consequently, it seems logical to think that each language mastered besides the mother tongue will construct, in parallel to the structure already put in place by the mother tongue, an additional structure, independent but intimately interconnected with the first, that will allow examining sensory perceptions and the neurolinguistic model of reality from an angle different from that afforded by the mother language, thus allowing use by the subject of considerably enriched and versatile neurolinguistic structures that will render conceptual thinking easier yet than if he mastered only his mother tongue. Isn't it a known fact that the greatest discoverers of the past in the fundamental sciences were generally polyglots?

\section{Objects and Their Characteristics}

Before proceeding to the description of the generalization mode by definition, or 
inclusion, let us shed a closer look on the manner in which language describes at the general level the various aspects of objects and processes, whether concrete or abstract.

We observe that the objects represented by our nonverbal perceptions are endowed with identifiable characteristics that allow us to describe them, that they are involved in progressive processes of changes of state of growth, degradation, color change, etc., that they are animated by intrinsic motion processes, such as rotating motions, inner oscillating motions, etc., that they are animated by relative motion processes with respect to each other, such as relative accelerations, relative resonance processes, etc.

We can therefore divide these characteristics into two very distinct categories: the static characteristics, that describe the states of these object at any given moment, and the dynamic characteristics, that are in fact the processes that describe the changes of state of these characteristics between a given moment and the following moment considered. In other words, static characteristics describe objects in space at a given moment, while dynamic characteristics describe them as they evolve over the course of time.

The static characteristics of objects are their colors, textures, shapes, dimensions, internal composition, etc., and their dynamic characteristics refer to any modification of their static characteristics, and to any motion of whatever nature that they could be animated by, intrinsically or relative to other objects.

\section{Verbal Representation of Observed or Idealized Objects and Processes}

Let us now give a closer look to these words that we use and that excite to the level of active awareness all of these arborescences of mental images. Making abstraction of the standard linguistic grammatical definitions usually associated to the different types of words, let us consider them specifically in relation with their neurolinguistic function.

The only types of words really required to finely tune the activation of the arborescences of nonverbal mental images in context with a speaker's whishes are the adjectives, the nouns, the verbs and the adverbs, or their replacing verbal locutions in case of absence or ignorance of a specific word in the articulated language of the speaker. All other types of words are simple connecting words imposed by the very variable syntaxes of the various articulated languages, meant to harmoniously relate the four types of keywords to correctly formulate an idea.

\section{The Function of Adjectives}

The word "adjective" is the name given to static characteristics of objects and processes, which are the qualities that we associate with them, that is, colors, shapes... white, black, round, long, tall, small, etc.

Within the neurolinguistic context, adjectives are, for all intents and purposes, the most important elements of all articulated languages, since they directly 
name the non-verbal characteristics of objects or processes. They thus constitute the lowest level of the structure of verbal description of the nonverbal images generated by our sensory perceptions, and of the non-verbal idealized nonverbal abstract concepts that develop as a consequence of the generalization process. In other words, they constitute the first level of abstract description of our non-verbal perceptions and so constitute the "bridge" between the thinking mode by images association and the thinking mode by words association. They thus establish the junction between the first and the second system of signalization.

It is also from adjectives that a great number of nouns, of verbs and adverbs are derived, and for good cause, since these nouns exist to name them while verbs and adverbs exist to allow us to think and speak about the states or changes of states of the characteristics identified by these adjectives.

When we describe an object or a process, we metaphorically bring to mind the list of its characteristics, which means that we strengthen to active awareness level the complete arborescence of its nonverbal characteristics that we have become aware of, that is, the list of the characteristics that allow us to distinguish it from all other objects or processes.

When using a group of words instead of a single word to describe one characteristic of an object or process, we are using a "verbal locution". This includes locutions that usage has standardized as well as locutions that we build on the spur of the moment, when thinking of the finer subtleties of characteristics of objects or processes that we are observing.

This later type of verbal label is particularly important, because we do not necessary always have at our disposal the exact word that would clearly describe a given characteristic of an object or process that we never thought about previously, or even to describe something that we already know, but the exact word for which momentarily escapes us.

These combinations of words that we assemble on the spur of the moment to describe shades of meaning that we formulate for the first time, often are our only tool to even think about these particular subtleties as we are trying to be a little more specific about some aspect of what we are describing. We consequently use verbal locutions each time that existing adjectives do not allow clearly representing a particular subtlety of a characteristic that we observe and want to describe.

\section{The Function of Nouns}

From the neurolinguistic perspective, a "noun" simply is the name, or title, of the list of characteristics that we associate to an object, a process, an action, a feeling, a quality, an idea, an abstract concept, etc. and that allows us to identify it. In other words, it is the anchoring point in the neocortex of the whole set of synaptic connections relating to this noun the synaptic imprints of all non-verbal characteristics that the person ever associated with the object, process, etc., that 
we refer to with this noun, and that constitute the associated arborescence, but will typically activate only the subset in this arborescence of such characteristics that the context of its use conjures up, at least those whose synaptic connections have not become too faint with the passage of time.

Consequently, from the neurolinguistic viewpoint, any word can become a noun as soon as we attempt to understand the idea that it conveys, that is, as soon as we attempt to identify the set of characteristics that we have associated to it. For collective intelligibility, of course, the idea expressed by a word is deemed to usually match the definition given in a dictionary.

However, what we typically understand of the idea expressed by a noun depends only on the specific list of static and dynamic characteristics that we associate to this noun in the context within which it is used. This means that the same noun used in exactly the same manner, but in different contexts, may refer to entirely different things, which could have absolutely nothing to do with the formal meaning of the word. This is the case of usage of any word as a figure of speech, or of its use in metaphorical context.

Of course, locutions can also be used instead of single word nouns to name an object or a process. What is a noun in fact, if not a summary of its definition? When we think of a new concept, of a new shade of meaning, or of a specific subtlety of a characteristic of an object, there may not even be a single noun that could directly describe what we are attempting to describe. In these cases, we have no option other than to think of it using its definition, so to speak.

\section{The Function of Verbs}

With the exception of "state" verbs, related to spatial perception, such as to be and to have, as well as many others, that refer to the presence or absence of a characteristic of an object or process, verbs always describe processes, which are related to time perception, that is, changes of state in space of the characteristics of objects and processes over periods of time, and for this reason they are named dynamic verbs, or action verbs.

State verbs allow associating a characteristic to an object or a process, or to dissociate it. A few examples: The flower is red. The flower is not red. The growth of the flower is rapid. The growth of the flower is slow.

Dissociation can take on the form of the negation of association, as was just shown, or of the replacement of a characteristic previously assigned by the assignation of a characteristic inherently incompatible. A few examples: Establish that a flower is white after having previously thought or established that it was red. Establish that its growth is stopped (has become static) after having thought or established that it was rapid or slow.

On their side, dynamic verbs allow associating increases or decreases in intensity of the characteristics of objects or processes. A few examples: The sky is darkening. The curtain is yellowing. The growth velocity is increasing (velocity being a characteristic, that is, a property of the growth process). Or: The growth 
process is slowing down (the velocity characteristic is implicitly assumed to exist).

In languages whose grammatical structure allows it, dynamic verbs indicating a change of state are often constructed from the adjective that names a nonverbal characteristic in process of changing; for example: white-whiten, dark-darken, etc.

If the grammatical rules of the language do not allow such a conversion for a given characteristic, a special verb, or one of its equivalents, can be used to describe the process, that is, to become. Example: The sky is becoming violet, or: The sky is turning violet.

In some languages, specific dynamic verbs exist to describe intrinsic cyclic continuous rotating and oscillating motions of objects, while in others, such intrinsic continuous cyclic motion have to be described with verbal locutions.

In English for example, two dynamic verbs exist to describe continuous cyclic rotating motion, "to rotate" and "to spin", while in French, a verbal locution must be used to verbally describe this motion, that is, "tourner sur soi-même-to turn about self", because the French dynamic verb "tourner" does not imply that the motion is cyclic.

In the case of dynamic verbs meant to describe relative motion of objects with respect to each other, the situation is reversed between English and French. While the verbs "approcher" and "éloigner" are available in French, verbal constructs such as "to close in" and "to move away" must be used.

\section{The Function of Adverbs}

Adverbs are words that can be joined to an adjective, a noun, a verb, or another adverb in order to modify or fine tune its meaning. They allow finely describing the degree of relative intensity or relative duration of nonverbal characteristics of the objects or processes that we observe or abstractedly conceive of. Just like verbs, many adverbs are constructed from adjectives. Example: He walks heavily. He walks happily, etc.

Obviously, adverbial locutions will be groups of words equivalent to adverbs that can be used each time existing adverbs do not clearly express the shade of relative intensity of a characteristic of an object or of a process that we would like to express. Example: Come here as soon as you can. I tried in vain to talk to him, etc.

In fact, in all circumstances, if a specific word is not available in a given language to represent a characteristic, an object or a process, some locution that can always be constructed in context on the spur of the moment to describe it.

\section{The Function of Inclusions in Sentences}

Starting from the premise that a word would be a summary of its definition, to get down to the individual characteristics of a concept defined by any given keyword, it would seem logical to think that if each keyword of this definition was resolved to its own definition, and so on for each keyword of these second 
level definitions, until the most detailed level is reached, at some point of the process, the whole set of nonverbal elementary characteristics of the first level initial word could be put to light.

But without going that far in directly clarifying the various sub-aspects of any idea being formulated, inserting this type of inclusions in our sentences is natural to us on account of the very nature of the structure associative by inclusion of any synaptic arborescence activated by the use of articulated language in the description and organization of our nonverbal perceptions. Example: Cats, which are very friendly animals, and that children love, not even mentioning adults, are mammals.

In such a voluntarily exaggerated sentence made up as an example, it could be assumed that what may have been initially meant to be said could have been only "Cats are mammals", but the mere mention of the word "cat" bringing to the level of active awareness the whole set of its characteristics during the process of formulating this idea, any one of these characteristics shared in common with other ideas could have caused the speaker to clarify by inclusion, on the spur of the moment, other aspects of our relation with cats, as could have happened during a conversation as a function of the context, the whole sentence remaining easily understandable while raising to active awareness level other aspects indirectly related to the first level idea being formulated.

\section{Verbal Communication, Oral or Written}

As previously mentioned, words convey the meaning that whoever hears or reads them associates with them, for the simple reason that they cannot activate in their respective brains the synaptic arborescence that the speaker or writer was actively aware of in his own brain as he was speaking or writing these words, but only the synaptic arborescences that each individual listener or reader associates to them by default. So if the speaker/writer is not careful in clearly describing what aspects of his own arborescence are involved, his message may not be correctly understood. This problem becomes even more acute when someone transmits the words of someone else to third parties.

In reality, we all are experts at naming, or labeling, things and concepts with nouns, properly using these nouns in various contexts afterwards, but often neglecting to let their full meaning rise to our active awareness, using the words as if they always were inherently self-explanatory.

In fact, as we learn words while growing up, they are of course self-explanatory for our own individual needs, since we personally define or accept the meaning of each of them. As we grow up, we constantly fine tune the meaning of each word we use with the help of parents, teachers, environment in general until we feel that we are sufficiently comfortable with articulated language, however subjectively, to be able to clearly express our needs and opinions, according to our personal vision of things.

For each of us, the names of object have simply become a series of "specific labels" that represent them and that we use to talk about them. When we stop to 
think of the meaning of a noun however, it metaphorically becomes a "first level label" activating a set of specific sub-labels, because its evocation raises to the level of our active awareness the whole series of second level other names that describe its various characteristics.

This notion of "first level label" that structures vertically, so to speak, the whole set of characteristics and sub-characteristics that allow describing an object or a process, also allows becoming aware that all of these first level names of object and processes can also be associated horizontally, so to speak, by similarity of the objects or processes themselves, but also by similarity of one of more of their characteristics, in all sorts of sets and categories that we identify with "generalizing labels".

This is what brings to light the direct parallel that exists between the highly abstract neurolinguistic structure associative by inclusion that allows coherent conceptual thinking, and the hierarchy of names associated by inclusion that were instrumental in establishing each of the synaptic imprints of which this arborescent structure is made of. This parallel will finally allow us to very concretely visualize the structure of the subjective neurolinguistic model of reality that each of us elaborates from birth onwards.

\section{A Specific Name Is a First Level Label Identifying a Single Object}

To clearly represent the difference between a "first level label" and a "generalizing labep", let us build a concrete example. If someone says "Yesterday, I practiced a Kung Fu move", he is not naming a specific move, but is indirectly alluding to a Kung Fu move not clearly defined and nobody will understand clearly what specific move is being referred to. This is a case where a generalizing label is being used, that is, a generalized term, or generalization.

If someone says "Yesterday, I practiced my Eagle claw push-up move", he is naming a specific Kung Fu move and an arborescence of clear nonverbal mental images will be raised to active awareness in the brain of anyone who knows Kung Fu. It does not matter if the set of personal images associated to the concept by each person familiar with Kung Fu is different. All that matters is that the structure of the concept represented by the mental images be preserved and that everyone identifies the same Kung Fu move.

For people unfamiliar with Kung $\mathrm{Fu}$, the same sentence will cause two unrelated arborescences to come up to active awareness, one for the "practice push-up move" first level label and one for the "eagle-claw" first level label. In context of a conversation about martial arts, some may possibly "figure out" the possible link between both concepts and tentatively end up with a mental image similar to that conjured up in the mind of Kung Fu practitioners, but for most, the sentence could remain unintelligible.

It should be easy now to perceive that the "Kung Fu move" label is twice removed from the actual physical event being referred to, "eagle claw push up" being the first level verbal description of the non-verbal actual move being de- 
scribed, that belongs by inclusion to the second level generalizing category "Kung Fu moves", and that activates the related arborescence.

\section{Hierarchies of Generalizing Labels}

All sorts of hierarchies of verbal generalization levels can be elaborated starting from a first level label. For example a person saying the aforementioned sentence about a Kung Fu move may have had in mind the generalized context presented in Table 1.

But somebody hearing this sentence may well understand it considering a generalizing context much less innocuous if he was a trained military, for example, as proposed in Table 2.

Or if another person hearing this same mention thinks in context that the speaker is boasting, presumably to make a good impression in front of strangers, might well consider an entirely different generalizing hierarchy, such as the one proposed in Table 3.

Table 1. Hierarchy of generalizing labels defining the context in which a person might be verbally using the "Eagle claw push up" first level label.

\begin{tabular}{cc}
\hline & Generalizing Hierarchy \\
\hline Generalization Level & Label \\
\hline Specific label & Eagle claw push up \\
$1^{\text {st }}$ & Kung Fu moves \\
$2^{\text {nd }}$ & Kung Fu \\
$3^{\text {rd }}$ & Martial Arts \\
$4^{\text {th }}$ & Sports \\
$5^{\text {th }}$ & Recreational activities \\
etc. & $\ldots$ \\
\hline
\end{tabular}

Table 2. Different hierarchy of generalizing labels defining the context in which another person hearing the same first level label might understand it.

\begin{tabular}{cc}
\hline \multicolumn{2}{c}{ Generalizing Hierarchy } \\
\hline Generalization Level & Label \\
\hline Specific label & Eagle claw push up \\
$1^{\text {st }}$ & Kung Fu moves \\
$2^{\text {nd }}$ & Kung Fu \\
$3^{\text {rd }}$ & Martial Arts \\
$4^{\text {th }}$ & Military training activity \\
etc. & $\ldots$ \\
\hline
\end{tabular}


Table 3. Different hierarchy of generalizing labels defining the context in which another person hearing the same first level label might understand it.

\begin{tabular}{cc}
\hline \multicolumn{2}{c}{ Generalizing hierarchy } \\
\hline Generalization Level & Label \\
\hline Specific label & Eagle claw push up \\
$1^{\text {st }}$ & Kung Fu move \\
$2^{\text {nd }}$ & Person possibly boasting \\
$3^{\text {rd }}$ & Insecure person \\
$4^{\text {th }}$ & Person not to be entirely trusted \\
etc. & $\ldots$ \\
\hline
\end{tabular}

Looking at the generalizing hierarchies just established, it can be observed that each level is included in the preceding levels in the decreasing order and that the first level label "eagle claw push-up" is implicitly included in each of the more general levels of each hierarchy, up to the highest level "recreational activities" label, the "military training activities" label or any other more general level that could have been activated in the brains of as many different persons who could have heard this sentence.

\section{Two Sorts of Labels: First Level and Generalizations}

In these hierarchies, only two types of labels can be identified, that is, a first level label that directly activates the nonverbal synaptic arborescence describing a specific object, and various generalizing labels defining various degrees of generalization activating no specific arborescence, but all of which somehow include the first level label mentioned, as well as an indefinite number of other possible first level labels, and that could consequently be defined as generalizations by definition.

Each of the generalizing labels refers by definition to more than one object and when used instead of a first level label, is likely to activate in the brain of each person hearing or reading it, the first level label that each person prefers or dislikes the most in the collection of cases that each would have associated to each of these generalizing label and would ultimately activate the corresponding arborescence.

For example, the generalized sentence "I ate an apple" will activate the arborescence of the nonverbal looks, textures and tastes of a Macintosh in the brain of people who prefer this variety, but will alternately activate an arborescence corresponding to some other variety for other people.

Alternately, the sentence " $I$ ate a Macintosh apple" will activate the arborescence corresponding to a Macintosh in the brains of every person who previously tasted this variety of apple, but will still activate the arborescence corresponding to some other variety for persons who never saw, manipulated or tasted a Macintosh, and no specific arborescence for persons who never saw any sort of apples. 


\section{What Are the Implications?}

We just observed how even first level generalizations can be confusing when the intention is to communicate information about a specific physically observable object for which an arborescence involving nonverbal sensory perceptions literally conjures up "images" mentally observable by imagination, but that only first level names can activate, and this, only in people who have previously experienced similar sensory perceptions.

The process of communication becomes even more complex when wishing to convey abstract ideas or concepts, because by nature, the verbal labels conveying abstract ideas or concepts do not intrinsically refer to sensory perceptions and consequently do not naturally activate mental images observable by imagination, despite the fact that such verbal descriptions seem at first glance to be the only means at our disposal to communicate abstract ideas or concepts.

An expression such as "comprehension process", for example, even though apparently a perfectly legitimate first level label, describes a non-verbal "abstract concept" instead of a physically observable nonverbal event such as the first level label "eagle claw push up". Let us thus assume for starters that it could be a first level generalization. Let us analyze where such an "abstract concept" stands in a generalizing hierarchy.

We see here four names that at first glance could mean just about anything. None of them seems to have a precise meaning, not even the expression that at first glance should logically be a first level label, that is, "comprehension process".

Being an abstract concept with no image being naturally associated with it, the next step in trying to activate a meaningful arborescence in the neocortex seems to be to establish its "extended definition". Table 5 thus tentatively proposes extended definitions for all levels of Table 4 generalizing hierarchy.

Considering this new tentative version of the hierarchy, we observe that the four abstract concepts extended definitions suddenly become significant. $\mathrm{Nu}$ merous readers may disagree with some of these definitions. This is due to the fact that each person tends to associate his own subjective verbal definition to any abstract concept, and that even a clear definition of an abstract concept is also a generalization. These various personal definitions are thus the true first level labels, in cases of abstract concepts.

Table 4. Generalizing hierarchy for an abstract concept.

\begin{tabular}{cc}
\hline \multicolumn{2}{c}{ Generalizing hierarchy } \\
\hline Generalization Level & Label \\
\hline $1^{\text {st }}$ & Comprehension process \\
$2^{\text {nd }}$ & Method \\
$3^{\text {rd }}$ & Methodology \\
$4^{\text {th }}$ & Theory of knowledge (epistemology) \\
\hline
\end{tabular}


Table 5. Generalizing hierarchy for abstract concepts including extended definitions for each generalization level.

\begin{tabular}{|c|c|c|}
\hline \multicolumn{3}{|r|}{ Generalizing hierarchy } \\
\hline Generalization Level & Label & Extended definition \\
\hline $1^{\text {st }}$ & Comprehension process & $\begin{array}{l}\text { A method used by the human brain, consisting in exploring and re-exploring a concept } \\
\text { until objective understanding of an object, event, process, or abstract concept has been } \\
\text { reached. }\end{array}$ \\
\hline $2^{\text {nd }}$ & Method & $\begin{array}{l}\text { Manner of saying, doing, following certain principles, according to a certain order, in } \\
\text { order to reach a certain goal. }\end{array}$ \\
\hline $3^{\text {rd }}$ & Methodology & Science whose object is the study of the methods of the various sciences. \\
\hline $4^{\text {th }}$ & Theory of knowledge & $\begin{array}{l}\text { 1) Science whose object is to evaluate the methods used in the various sciences in their } \\
\text { relations to philosophy. } \\
\text { 2) Science whose object is to define a universal method for understanding reality in its } \\
\text { widest sense. }\end{array}$ \\
\hline
\end{tabular}

Granted that for any abstract concept, all possible first level definitions seem to be subjective; this does not mean that one specific first level definition cannot exist to be met with general collective approval. Indeed, such collectively agreed upon specific definitions of abstract concepts is mandated for intelligible mutual communication in any community. These formally established first level definitions are available in dictionaries and in the notes of the conferences during which they were established.

Considering Table 5 again, we observe that since label "comprehension process" can potentially correspond to many definitions, it cannot possibly be a first level label, and simple analysis rapidly reveals that the definition itself of the generalizing label "comprehension process" is the first level label of this abstract concept. Let us now set up the final form of the generalizing hierarchy presented in Table 6, taking this conclusion in to account.

So the major conclusion emerging from this analysis is that the extended definition of an abstract concept is in reality its first level verbal description, which is the verbal description of the nonverbal concept itself. We thus observe that just like articulated language is the only means at our disposal to describe nonverbal objects, processes and events perceived from our sensory perceptions, it also is our only means to describe the idealized abstract nonverbal objects, processes and concepts synthesized from the generalizations emerging from the use of language itself.

One can observe by examining the extended first level definitions of Table 6 that although these definitions are easily understandable, they do not automatically activate an arborescence of "images" in the brain, as the first level label "eagle claw push up" automatically does. The reason is that contrary to the first level names of the nonverbal "images" of objects originating by structure from our sensory perceptions, that thus preexist in our memory before they can be observed and named, the extended description of abstract objects stemming from the generalization process may not always coincide with a corresponding 
Table 6. Generalizing hierarchy for abstract concepts including identification of the first level label.

\begin{tabular}{cll}
\hline Generalization Level & \multicolumn{1}{c}{ General Label } & \multicolumn{1}{c}{ First level label } \\
\hline & Comprehension process & $\begin{array}{l}\text { A method used by the human brain, consisting in exploring and re-exploring a } \\
\text { concept until objective understanding of an object, event, process, or abstract } \\
\text { concept has been reached. }\end{array}$ \\
$1^{\text {st }}$ & Method & $\begin{array}{l}\text { Manner of saying, doing, following certain principles, according to a certain order, } \\
\text { in order to reach a certain goal. }\end{array}$ \\
$3^{\text {rd }}$ & $\begin{array}{l}\text { Science whose object is the study of the methods of the various sciences. } \\
4^{\text {th }}\end{array}$ & $\begin{array}{l}\text { 1) Science whose object is to evaluate the methods used in the various sciences in } \\
\text { their relations to philosophy. } \\
\text { 2) Science whose object is to define a universal method for understanding reality in } \\
\text { its widest sense. }\end{array}$ \\
\hline
\end{tabular}

pre-synthesized abstract image, given that these idealized images can only be conceived from the verbal generalizing process itself.

They must then be conceptualized and described before they can be "imaged", so to speak. We give to these constructed images that we build to correspond to abstract concepts names such as "graphical representations", "symbolic representations" or simply "symbols". It is this very process which is at the origin of the mathematical thinking mode that we will soon analyze, and that constitute our third system of signalization.

These symbols can be voluntarily elaborated or chosen, but the neocortex is perfectly capable of integrating to a high degree a concept clearly defined verbally to eventually generate a nonverbal symbolic representation and integrate it to a person's thinking mode by images. The well known representation of the "gravity wells" which give a visual representation of the effects of gravity in the Theory of General Relativity is such a graphical representation of an abstract concept. The bell shaped IQ curve is another.

Just like we have a natural tendency to name the objects, processes and events whose images are automatically stored in our brain as a result of our sensory perceptions, we also have a natural tendency to build graphical representations in order to associate images with the abstract concepts that we elaborate by logical association of the generalizations stemming from the use of an articulated language.

Whenever we read or listen to the verbal description of a new abstract object or concept, even after having clearly understood and accepted its extended verbal description as adequate, if a corresponding image is not provided at the same time, we tend to remain uneasy and thoughtful for a while with such a new concept. This period of uneasiness seems to coincide with the time required for a satisfying symbolic representation to be built and integrated in the nonverbal areas of the neocortex; this operation allowing the complete integration of the new abstract concept in our conceptual thought processes. 


\section{Cogitation and Conversations by Correlation of Generalizations}

Unless involving an emotionally loaded issue or one that elicits any level of uneasiness as previously analyzed, the process of verbal cogitation and exchange of ideas during conversations will not involve the limbic system and will remain a process internal to the neocortex. Cogitation, or conceptual thinking, simply amounts to silent internal conversation with self, involving the very same mental processes as a verbal conversation with another person, except that the speech organs and writing praxis are not used. Chauchard used to refer to conceptual thinking as the "interior language".

"Humans do not speak because they think, they speak because their thinking process is an interior language, a human means of thinking. Language is not at the service of the thinking process, it is first and foremost this thinking process and then its communication. To think, is to associate cerebral images (giving here to the word 'image' a general meaning extending beyond mere sight) that education creates within us from our sensory perceptions that can be conjured up through imagination, and that have thus become an interior code, an autonomous manner with which we can use our brain. To each image is substituted through education its verbal denomination. Since they name everything, instead of associating images, human beings can directly associate the corresponding names, a system more adequate to fully extend the abstraction capabilities of the human brain (Chauchard, 1960b: p. 122)".

Cogitation and conversations are entirely grounded on the generalization process that allows an unequalled flexibility of the thinking process. Unsurprisingly, the generalization process is ruled by the same four comparison activities that have been identified as the natural association and correlation properties identified as sustaining the learning process, that is, association, classification, discrimination and evaluation. As previously mentioned, the first three are correlation functions, that is, association, classification and discrimination of the elements to be considered, that associate these elements according to four fundamental comparison criteria, which are simultaneity, successively, similarity and dissimilarity (Blayo \& Verleysen, 1996: p. 23).

The flexibility of the generalization process is due to the fact that every word of articulated languages can have an extension of meaning going from the object individually identified, that causes the related arborescence to surge to the level of active awareness, up to its more general sense in context, also allowing all intermediate generalization levels, and this, strictly as a function of the context of their use.

If I say "I like this pencip", I obviously refer to a single object and the word pencil becomes a first level label used to identify a single object. If I say "The pencil is a useful tool for writing", I just as obviously refer here to pencils in general, and the word pencil, despite being used in the singular mode, becomes a generalized label used in a much more general sense. We can easily conceive of a 
context where the word pencil will refer only to the pencils present in a specific room, or to those present in the same building, or to all pencils that ever existed.

When cogitating on issues that do not activate the limbic system, the fourth activity sustaining the learning process, that is evaluation, which is primarily controlled by default by the limbic system, is now left under the complete control of the actively aware will of the subject. The extent of meaning of each word attached to each word of a sentence is then determined uniquely by the context, and the correlation between the words thus defined by a complete sentence ensures that the meaning of this sentence causes a narrowing of the context that will influence the meaning of the words of the following sentences, progressively setting up the stage to drawing conclusions whose logic seems inescapable. All informal conversations occur according to this mode.

"This active awareness is not a passive spectator, it intervenes actively to control the cerebral activity, recall images and elicit actions... The characteristic of the normal human brain, thanks to its complexity, is to be the organ that allows freedom. Active awareness manifests itself as a narrow beam that illuminates only a small part of the cerebral functioning. It appears as a state of hypervigilance involving an excitation of what we observe and an inhibition of what we are not paying attention to, according to autoregulating processes that have their seat in the reticular formation" (Chauchard, 1960b: p. 127).

On the other hand, for utterly strict formal conclusions to be logically drawn, as in scientific articles of all natures, the progressive narrowing of the context leading to inescapable conclusions on issues of great complexity must be guided with the highest level of precision. This level of thoroughness is reached when the characteristics of each individual object which is part of each set defined by the generalizing labels is examined to ensure that each object rightly belongs to the set.

In practice, such an examination involves a "vertical" search, so to speak, in the sets of characteristics of each of the arborescences activated by the first level labels of each object belonging to a generalization by definition. The more numerous will the characteristics identified as being common to the various arborescences belonging to the set generalized by definition be, the more tightly knit will the "horizontal" correlation be, so to speak, between the objects of the set.

The label "human body", for example, will activate a much less elaborate arborescence in the mind of a non-specialist than in the mind of a surgeon, who will elaborate vastly more complex generalized subsets on this issue while conversing with another surgeon than could a non-specialist discussing the same issue with another person, which incidentally highlights the importance of the extent of knowledge in drawing formally valid conclusions. The list of characteristics common to all objects of a set then constitutes the formal reference frame of each set generalized by definition.

In a context of formal research, it is not prudent to assume that all of the objects of a set defined by definition at first glance really are part of this set. For 
example, it was current even in 2018 in the physics community to think that atoms are made of three elementary particles that would be the electron, the proton and the neutron, and this, even in numerous quite formal reference works (Giancoli, 2008; Sears, Zemansky, \& Young, 1982); while it had been experimentally demonstrated exactly 50 years before that the proton and the neutron are not such elementary particles, but are rather systems of elementary particles themselves, just like the Solar system is not a heavenly body, but a system of heavenly bodies.

Indeed, the great Stanford Linear Accelerator (SLAC) was built specifically in the 1960's to become able to accelerate electrons with sufficient energy to penetrate and explore their structure by non-destructive scattering. That's how two more truly elementary particles were discovered making up their internal structure, that are of the very same electromagnetic nature as the electron, and that de facto constitute with the electron the true set of three elementary particles of which all atoms are made (Breidenbach et al., 1969; Michaud, 2013a; Michaud, 2017c).

The question that now comes to mind is then the following: what further progress could already have been accomplished in fundamental research if this discovery had immediately been clearly updated in all reference works, so that all physicists and mathematicians had had it in mind these past 50 years?

\section{The Mathematical Thinking Mode}

As put in perspective in the introduction of this paper, over the course of history, a very special set of nonverbal abstract concepts and symbols emerged from the use of articulated language, that is, the idealized geometric concepts resulting from our perceptions of the shapes of objects in our environment, and the abstract mathematical concepts resulting from our perception of the distances between objects and of the changes in their characteristics in the course of time, that provide us with usable tools to measure all aspects of these spatial distances and time related changes.

Contrary to the arborescences of images that each person associates to his sensory perceptions, and that nothing allows to verify whether they are identical for all individuals, it is easy to verify that the abstract idealized geometric images resulting from the generalization of the shapes perceived in nature, such as the circle, the sphere, and all other geometric shapes, are perceived in an identical manner by everybody once conceptualized and verbally described, and the same identity of perception can be confirmed for all abstract mathematical concepts.

Over the course of time, thus emerged a universally intelligible nonverbal symbolic language combined with a set of idealized geometric forms that allow discussing and easily exchanging ideas about the nature of the objects and processes observed in our environment, irrespective of the differences in the mother tongues of the people involved; so intelligible in fact, that carefully prepared mathematical derivations accompanied by appropriate idealized graphical 
illustrations can be understood by any mathematician even if the accompanying comments are written in an unknown articulated language.

Numerous people take interest in mathematics and learn to master this nonverbal symbolic language to the point of becoming able to use it directly to conceptually think in this idealized and generalized manner about the processes that are suspected of objectively existing in our environment as well as about the nature and characteristics of the objects involved in these processes (Howell \& Bradley, 2001), mentally reproducing, so to speak, in an idealized and measurable manner, the objects, processes and events observed in the real environment.

Since this abstract conceptual language develops in neocortex areas different (Amalric \& Dehaene, 2016) from those that are the seat of the thinking mode by nonverbal images stemming from our sensory perceptions, identified by Pavlov as the first system of signalization, and the verbal areas, seat of verbal thinking mode, identified as the second system of signalization; the nonverbal mathematical language that develops by education in these other areas can be considered as being a "third system of signalization", which is universally intelligible by all who learn to master it.

The mathematical language constitutes the most advanced form of language that we possess. It has been freed from any requirement to directly refer to our sensory perceptions and allows us to study, describe and measure by imagination the relationships between idealized representations of the objects that physically exist in our environment.

In fact, these idealized geometric representations allow us to generalize graphically the characteristics that are common to these physically existing bodies. It is not possible to retrace historically the exact origin of the geometric concept of the circle, for example, but it is easy to speculate that the shape of the full moon, visible since before our species appeared, might well be its origin. But irrespective of this origin, once the idea of the circle was begotten, it is not difficult to conceive that observation of its shape by imagination could have eventually led to understand that the line that delimits its circumference is everywhere equidistant from its center, for example.

There is no doubt that all of the other properties of the circle and of all other idealized geometric shapes were discovered by means of similar observations and by the same mechanisms of cogitation that allow us to discover the properties of objects identified in our environment from our sensory perceptions. Euclid's theorems augmented by the complementary concepts formulated by Lobatchevski, Riemann and many others constitute the sum of the idealized geometric concepts in current use.

If we now imagine a straight line passing through the center of this idealized circle, thus defining an axis of rotation, and that we imagine that the circle rotates 180 degrees about this axis, we can visualize by imagination that its motion defines a sphere that now circumscribes a volume of space under a surface, all points of which also are at equal distances from this center. Even readers who 
did not have the opportunity in the past of cogitating about this issue now have a mental image of the idealized sphere that all mathematicians are using.

An invariant relation was then discovered about this idealized sphere, involving the distance between its center and its surface, named the radius $(r)$, allowing, if we know its length, to precisely calculate the circumference, the surface area and the volume of this sphere, which is mathematically defined as a volume of space circumscribed by a closed surface all points of which are located at equal distances from the center of its volume, where $\pi$ is the invariant relation expressed by the number of times that the diameter $(d)$ of the sphere (twice the radius, or $d=2 r$ ) is contained in the circumference $\pi=3.141592654$. This is how the first mathematical equations were established to measure the length of the circumference $(C)$, surface area $(A)$ and volume $(V)$ of this idealized geometric sphere.

$$
C=2 \pi r, \quad A=4 \pi r^{2}, \quad V=\frac{4}{3} \pi r^{3}
$$

The question that comes up at his point, is how can the calculation means of circumference, surface area and volume of this idealized sphere be transposed to measure physically existing objects, because it is well understood that no real object is as perfectly spherical in our environment?

In the astronomical domain, it is well known that the Earth, for example, is not a perfect sphere, but rather a sphere slightly flattened at the poles, due to its rotation velocity, with a polar radius estimated at $6357 \mathrm{~km}$ and an equatorial radius estimated at 6378 , that is, a difference of $21 \mathrm{~km}$, not even speaking of the irregularities of its surface, the largest of which are the Everest rising to $8.8 \mathrm{~km}$ above the surface and the Mariana trench sinking by close to $11 \mathrm{~km}$ below the surface.

What radius should be chosen then? The simple method is to calculate a mean radius that would correspond to that which the Earth would have if it became a perfect sphere. This mean radius has been calculated as being $6371 \mathrm{~km}$. This radius is calculated by considering the center of mass of the Earth as the center of this sphere. The center of mass of a body is the unique dimensionless point about which this body could be in rotation irrespective of the orientation in its mass or of its axis of rotation (Giancoli, 2008). The same technique can be applied to absolutely all existing celestial bodies whose forms are detectable. For celestial bodies too far away for them to be detected directly otherwise than as a single luminous point without any measurable dimensions, or indirectly by interaction with other bodies, the spherical shape is assumed for calculation purposes.

All sorts of similar mathematical techniques have been developed to harmonize the idealized mathematical methods with the real characteristics of objects that physically exist in our environment. They are available in numerous reference works meant for engineers of all specialties, such as the monumental general physics work of Douglas Giancoli (Giancoli, 2008), and numerous more 
specialized reference works on thermodynamics, mechanics of materials, resonance phenomena, etc., some of which are mentioned here for convenient reference (Çengel \& Boles, 2002; Meriam \& Kraige, 2003; Rao, 2005; Hibbeler, 2005).

Another example of the usefulness of the idealized geometric concepts imagined thanks to the generalization process is Carl Friedrich Gauss's contribution in the $19^{\text {th }}$ century, precisely in relation with the concept of the idealized sphere already mentioned. He had the idea of exploring the mathematical possibilities offered by the concept of varying from zero length to infinite length of the radius of the idealized sphere previously defined by mathematician Leonhard Euler, which allows conceptually visualizing an infinite number of virtual concentric spheres, or alternately a single sphere whose volume varies omnidirectionally.

His genial idea was to associate this varying radius to the Coulomb force, that varies non-linearly with the inverse square of the distance between any pair of electrically charged particles, and whose equation is:

$$
F=\frac{e^{2}}{4 \pi \varepsilon_{0} r^{2}}
$$

Incidentally, one can observe in passing that Equation (1) to calculate the area of the idealized sphere $\left(A=4 \pi r^{2}\right)$ is an integral part of the divisor of the Coulomb Equation (2), and one could speculate that this may have been the detail that caused Gauss to associate the concept of the idealized sphere to the Coulomb equation. By removing one of the charges from Equation (2), he defined the concept of the electric field $(E)$, whose equation is the following:

$$
\boldsymbol{E}=\frac{e}{4 \pi \varepsilon_{0} r^{2}}
$$

The consequence is that Equation (3) allows visualizing by imagination a virtual force field of punctual infinite intensity at the location of the remaining charge, that could be an electron for example; an intensity that would reduce omnidirectionally as a function of the inverse square of the distance as the distance from this charge increases (Sears, Zemansky, \& Young, 1982: p. 480). One only needs to re-introduce a second charge anywhere in this virtual field to recuperate the Coulomb Equation (2) and calculate the real intensity of the force and the corresponding adiabatic kinetic energy induced in each charge (Michaud, 2018):

$$
F=e \boldsymbol{E}=\frac{e^{2}}{4 \pi \varepsilon_{0} r^{2}} \text { and } E=r F=\frac{e^{2}}{4 \pi \varepsilon_{0} r}
$$

It is this definition technique of electric and magnetic fields that allowed Maxwell to synthesize the equations of Gauss, Ampere and Faraday into a coherent whole that allows calculating the invariant speed of light in vacuum from their equations, which is one of the grounding elements of modern electrodynamics. Incidentally, the Gauss equation is now better known under the name of First Maxwell equation. 
It must also be mentioned at this point that contrary to the general impression, modern electrodynamics (Griffiths, 1999; Jackson, 1999) is not directly grounded on Maxwell's theory, that mandates that both the electric and the magnetic field of electromagnetic energy have to oscillate in alternance while mutually inducing each other for the energy to exist and propagate, but on Ludwig Lorenz's interpretation that implies that both fields oscillate synchronously (Cornille, 2003: p. 449), an interpretation with which Maxwell disagreed. The shade of meaning is important because Lorenz's interpretation does not allow harmonizing localized electromagnetic quanta with some aspects of electrodynamics, while Maxwell's allows complete harmonization when correlated with Louis de Broglie's conclusions (Michaud, 2016c).

These few examples of conceptualization of idealized geometric forms and corresponding mathematical equations show how useful they can be in measuring and calculating the various characteristics of the objects and processes that exist in our environment. The sum of the mathematical concepts elaborated to this day to calculate all aspects of the properties of objects and processes in our environment are available in multiple reference works some of which are given here in reference for convenience (Fankel, 1997; Hassani, 1999; Eisberg \& Resnick, 1985).

The book titled "Mathematical Physics" in particular, by Sadri Hassani (Hassani, 1999), provides a substantial historic summary of the contributions of most of the great mathematicians of the past. Another reference work also deserves to be mentioned in this perspective. It is a collection of essays by eminent mathematicians from a score of American universities covering various aspects of mathematics, edited by Russell Howell and James Bradley (Howell \& Bradley, 2001), replete with more references to other significant works on the nature of mathematics.

All of these idealized geometric and mathematical concepts are of such perfection that their inherent esthetic beauty easily causes a loss of sight of the fact that they are virtual and do not directly reflect physical reality. It is important to resist the idea that the virtual idealized fields defined by Gauss could physically exist, for example, since the mechanics of their idealized conception from verbal generalizations is the proof that they simply are part of the nonverbal mathematical language that we collectively developed to visualize by imagination an idealized version of the physical reality of our environment, in order to measure and understand it. Care must always be taken while using it in describing physical processes to take into account the physical limits that really exist.

A last point of interest remains to be put in perspective with regard to the mathematical equations themselves, because just like the words of articulated languages can define by context a single specific object or process that will activate the corresponding synaptic arborescence to the active awareness of the subject, or alternately define a generalization that activates no specific arborescence, the mathematical equations also are subject to a similar dynamics; first level eq- 
uations activating the corresponding arborescences of the idealized geometric forms to the level of active awareness and the generalized equations activating no specific arborescence. This means that the equations fill the same function with respect to the nonverbal idealized geometric concepts that the words of articulated languages fill with respect to sensory perceptions, and that metaphorically speaking, the equations are the "words" of the mathematical language.

This is what allows understanding why the active awareness of mathematicians can remain voluntarily concentrated for extended periods of time to cogitate exclusively on the alternate "idealized virtual environment" observable via the triad articulate-language/idealized-geometric-concepts/mathematical-equations as easily as they can remain alternately concentrated to cogitate exclusively on the "real environment" via the tandem articulate-languagel sensory-perceptions, or even in "virtual-real environments superposition", observable via the combined use of the quadruple set articulate-language/mathematical-equations/idealizedgeometric-concepts/sensory-perceptions, a state that may even have become permanent due to mathematical thinking mode training, as apparently demonstrated during the face recognition tests made during the Amalric-Dehaene experiments (Amalric \& Dehaene, 2016), which is a condition that definitely deserves to be further investigated.

The junction between this idealized virtual environment and the real environment can truly be made only via the first level equations that directly activate the arborescences of the idealized geometric concepts, provided that they could be resolved with numerical values established by means of actual experiments in the real environment observable via our sensory perceptions. In the domain of fundamental physics, for example, this research has allowed identifying over the course of time about 200 of these numerical constants that are now available in reference works such as the CRC Handbook of Chemistry and Physics (Lide, 2003), and other sources for the various scientific domains.

For example, this constant research has allowed up to now to observe that all celestial bodies seem captive in stable least action resonance states at mean distances from central bodies, mean distances that are directly usable to solve the mathematical equations established for the idealized circular and spherical geometric shapes. These mean distances are located between precise stable minimal and maximal axial distances (perihelion and aphelion) from the central bodies. These three stable numeric values allow defining clear volumes of space being visited by each orbiting body over the course of time about the central bodies. Such volumes can evolve into rather complex shapes for bodies that have satellites, that induce interference beat patterns that modify the otherwise regular volumes that would be visited by a body without satellite. In fact, all bodies in any stabilized axial system somehow influence each other's trajectories and the shapes of the resonance volumes that they visit. A similar electromagnetic dynamics defined by quantum mechanics $(\mathrm{QM})$ is also applicable at the submicroscopic level to the elementary particles making up the atoms of which all ma- 
croscopic masses are made, including our own bodies (Michaud, 2018).

Effectively, solving first level equations by means of these experimentally verified values is indeed the only way to distinguish in the set of all equations, those that have a truly predictive value in the real environment by taking into account the physical limits that really exist, from those elaborated without taking these limits into account by virtue of the unlimited potential of the idealized geometric concepts, that by nature do not possess such inherent limits. The speed of light, for example, is confirmed as being the fastest velocity at which electromagnetic energy can move in the real environment, despite the fact that vastly superior velocities can easily be imagined in the virtual environment.

How to distinguish first level equations from generalized equations is the last issue remaining to be clarified. A few examples will allow shedding clear light on the difference. Let us take as an example one of the simplest while at the same time one of the most general equations of fundamental physics, that is, the equation that defines the force of acceleration $(F)$ that applies to any physically existing massive body, corresponding to Newton's second law:

$$
F=m a
$$

where " $m$ " represents a mass and " $a$ " represents an acceleration. The reason why it is possibly one of the most general force equations is that all classical force equations can be reversibly derived from it, including the Coulomb Equation (2) previously mentioned (Michaud, 2013b), which on its side is possibly the most important first level equation identified to date (Michaud, 2018: p. 1066). We will not enter into any relativistic considerations here to keep the demonstration simple.

In this equation, " $m$ " can represent any existing mass, which already causes no particular massive body to come to mind. If the equation is reformulated to represent the well known invariant rest mass of the electron, whose symbol is " $m_{e}$ ", the equation already gets nearer to the first level

$$
F=m_{e} a
$$

A first level symbol, such as " $m_{e}$ ", is a symbol that can be replaced in an equation either by one of the numerical physical constants already established (Lide, 2003), or by a variable numeric value belonging to a sequence possible in the real environment.

The case of symbol " $a$ " for the acceleration is more complex, because it needs to be replaced by two first level symbols to become significant, that is, a velocity " $V$ " and a distance " $d$ ". In the case of the hydrogen atom, the precise theoretical velocity corresponding to the real mean radial distance between the electron stabilized in a state of axial resonance with respect to the nucleus of the atom is well known and is represented by symbol " $V_{B}$ ", and is known as the theoretical velocity at the Bohr orbit, and the symbol of the radius of this theoretically perfect mean orbit is " $r_{B}$ ", and is known as the Bohr radius:

$$
a=\frac{v^{2}}{d}, \text { consequently: } F=m_{e} \frac{v_{B}^{2}}{r_{B}}
$$


The numerical values represented by first level symbols " $V_{B}$ " and " $r_{B}$ " belong in reality to sequences of velocities and distances that are physically possible in the real environment, but also are to be found in the list of fundamental physics constants (Lide, 2003) because they corresponds to very precise stable states in the real environment.

Equation (7) is now converted to a first level equation that will give a precise value to the force $(F)$ being physically applied between the electron and the hydrogen atom nucleus at the mean resonance distance that separates them, and will activate to the level of active awareness the whole arborescence of the knowledge that each physicist and mathematician will have personally accumulated about the electron and the hydrogen atom, including the much more complex Schrödinger equation that describes the resonance volume visited by the electron about the Bohr radius (Michaud, 2018), an equation that would be out of context to mention here, but which is available in a number of reference works (Giancoli, 2008; Eisberg \& Resnick, 1985) as well as on the Internet.

It is uniquely the activation of this type of arborescences to the level of active awareness that allows identifying the aspects of objective physical reality that remain to be clarified and orients research in promising directions.

Another complexity is added to the fact that Equation (5) is inherently general. It is the fact that there exists numerous ways to symbolize a mathematical equation, and that this fundamental equation, like many others, is often represented under a variety of forms, all valid as a function of the context of their use, but all being as general as Equation (5) and activating no arborescence associated to our sensory perceptions, of which here are a few examples, which all are alternate representations of Equation (5):

$$
F=m \cdot \frac{\partial^{2}}{\partial t^{2}} u(x+h, t), \quad \sum_{i} \boldsymbol{F}_{i}=\frac{\mathrm{d} \boldsymbol{p}}{\mathrm{d} t}, \quad \boldsymbol{F}=m \frac{\mathrm{d} \boldsymbol{v}}{\mathrm{d} t}, \text { etc. }
$$

These three forms were verified to be convertible to a first level equation such as Equation (7), but it is prudent to take on the habit of verifying in this manner all equations proposed without this verification in articles of whatever source. Such a habit has the additional benefit of improving synchronization between the three modes of thinking. Fortunately, all reference works meant for the engineering community, such as the few mentioned here (Giancoli, 2008; Çengel \& Boles, 2002; Meriam \& Kraige, 2003; Rao, 2005; Hibbeler, 2005) resolve all general equations to first level. Some textbooks meant for teaching are remarkably well made in this regard (Sears, Zemansky, \& Young, 1982; Eisberg \& Resnick, 1985).

Among others, Louis de Broglie was in the habit of always doing this verification, which occasionally reserves happy surprises, since it is in this manner that he discovered the relation that exists between Planck's constant and the precise amount of energy induced at the mean resonance distance of the electron in the hydrogen atom, as analyzed in a separate reference (Michaud, 2017c), and it is this same habit that allowed discovering, among other valuable surprises, that all 
classical force equations are variations of the Coulomb Equation (2) and of the fundamental acceleration Equation (5) (Michaud, 2013b), thus allowing the harmonization of classical/relativistic mechanics with electromagnetic mechanics (Michaud, 2017a).

\section{Conclusion}

It turns out that conceptual thinking operates in three different modes, that is, the non-verbal thinking mode by association of images naturally synthesized by the brain from sensory perceptions from the environment, which is of the same nature as the less performing thinking mode of species having developed less advanced nervous systems (Chauchard, 1960b), identified by Pavlov as the first system of signalization (Pickenhain, 1998); the verbal thinking mode by words association, acquired by education, which allows abstracting and generalizing the signals of the first system, identified by Pavlov as the second system of signalization; and finally, the mathematical thinking mode by idealized concepts association put in perspective in this study, resulting from the generalizations provided by the use of articulated languages, consisting in a set of collectively intelligible idealized geometric and mathematical concepts collectively defined and acquired by education, which can be identified as the third system of signalization, that enables the establishment of an idealized virtual collective model of the real environment, and that allows measuring and understanding it.

Three important conclusions can be drawn from this study. The first is the importance for each child to develop all verbal skills up to autonomy before the age of 7 . This conclusion can be drawn from the correlation between the works of Paul Flechsig (Flechsig, 1920), Paul Chauchard (Chauchard, 1960b) and Dolores Durkin, whose results are mentioned in the work of Fitzhugh Dodson (Dodson, 1971).

Indeed, the discovery by Flechsig of the genetically programmed delay in the myelination of the verbal areas of the neocortex until the age of 7 seems to directly correlate with Chauchard's observation that the density of the interconnected network being built in the verbal areas during articulated language acquisition is much denser in those of children who complete this learning before the age of 7 than in those of children who did not complete this learning in a timely manner, which seems to be directly related to the observation made by Dolores Durkin that all children who had learned to read before the age of 7 dominated markedly in all learning tasks over the entire period of their primary schooling in California with none of the children who had learned to read past this age being able to catch up with them. This correlation should therefore be studied further.

The same correlation can be made between the fact that in Finland, all children are schooled into mastering all verbal skills before the age of 7 , which directly explains why the illiteracy rate of the adult population is practically zero in this country, whereas in the province of Quebec, Canada, where the systematic 
teaching of verbal skills before the age of 7 was abandoned 50 years ago as part of an innovative schooling experiment, the rate of functional illiteracy of the adult population has gradually increased over time to the still growing level of $53 \%$ reached in 2013 according to the OECD figures, to the continued bafflement of the local experts (Michaud, 2016b), cutting this entire segment of the population from any access to the widening range of functional literacy dependent jobs, an area affected by a chronic lack of manpower in the same community.

The second conclusion that emerges from this study is that all of the researchs carried out over the past hundred years confirm that articulated language acquired by education in infancy is the central element of conceptual thinking, allowing the generalization of the knowledge acquired about physical objects and processes observed in the real environment from our sensory perceptions, as well as the generalization of the knowledge gained through observation of the pool of their collectively developed idealized representations in the form of a virtual environment; idealized thanks to the generalization ability provided by articulated language.

The third conclusion is that it is possible to closely synchronize the idealized collective virtual environment resulting from the generalizations provided by the verbal thinking mode with the real environment, by means of the mathematical thinking mode as analyzed in the previous section, to accurately measure and understand collectively all aspects of the real environment.

\section{Conflicts of Interest}

The author declares no conflicts of interest regarding the publication of this paper.

\section{References}

Amalric, M., \& Dehaene, S. (2016). Origins of the Brain Networks for Advanced Mathematics in Expert Mathematicians. Proceedings of the National Academy of Sciences of the United States of America, 113, 4909-4917.

http://www.unicog.org/publications/Amalric\%20Dehaene\%20fMRI\%20of\%20math\%2 0and\%20language $\% 20 \mathrm{in} \% 20$ professional\%20mathematicians\%20PNAS $\% 202016 \% 20 \mathrm{pl}$ us\%20SI.pdf https://doi.org/10.1073/pnas.1603205113

Anderson, J. A. (1995). An Introduction to Neural Networks. Cambridge, MA: The MIT Press.

Bergelson, E., \& Swingley, D. (2012). At 6-9 Months, Human Infants Know the Meanings of Many Common Nouns. Proceedings of the National Academy of Sciences of the United States of America, 109, 3253-3258. https://doi.org/10.1073/pnas.1113380109

Bickart, K. C., Dickerson, B. C., \& Feldman Barret, L. (2014). The Amygdala as a Hub in Brain Networks That Support Social Life. Neuropsychologia, 63, 235-248. https://www.sciencedirect.com/science/article/pii/S0028393214002760?via\%3Dihub https://doi.org/10.1016/j.neuropsychologia.2014.08.013

Blackbill, Y., \& Fitzgerald, H. E. (1972). Stereotype Temporal Conditioning in Infants. 
Psychophysiology, 9, 569-577.

https://onlinelibrary.wiley.com/doi/pdf/10.1111/j.1469-8986.1972.tb00766.x https://doi.org/10.1111/j.1469-8986.1972.tb00766.x

Blayo, F., \& Verleysen, M. (1996). Les réseaux de neurones artificiels, Presses Universitaires de France. Que sais-je? No. 3942. France.

Brannon, E. M., Suanda, S., \& Libertus, K. (2010). Temporal Discrimination Increases in Precision over Development and Parallels the Development of Numerosity Discrimination. Developmental Science, 10, 770-777.

https://www.ncbi.nlm.nih.gov/pmc/articles/PMC2918408/pdf/nihms213768.pdf https://doi.org/10.1111/j.1467-7687.2007.00635.x

Breidenbach, M. et al. (1969). Observed Behavior of Highly Inelastic Electron-Proton Scattering. Physics Review Letters, 23, 935-939.

http://www.slac.stanford.edu/pubs/slacpubs/0500/slac-pub-0650.pdf

https://doi.org/10.1103/PhysRevLett.23.935

Çengel, Y. A., \& Boles, M. A. (2002). Thermodynamics-An Engineering Approach. New York: McGraw-Hill.

Chauchard, P. (1944). Les messages de nos sens, Presses Universitaires de France. Que sais-je? No. 138. France.

Chauchard, P. (1960a). La chimie du cerveau, Presses Universitaires de France. Que sais-je? No. 94. France.

Chauchard, P. (1960b). Le cerveau et la conscience, Les éditions du Seuil, France.

Chauchard, P. (1963a). Le cerveau humain, Presses Universitaires de France. Que sais-je? No. 768. France.

Chauchard, P. (1963b). Physiologie de la conscience, Presses Universitaires de France. Que sais-je? No. 333. France.

Chauchard, P. (1970a). Le language et la pensée, Presses Universitaires de France. Que sais-je? No. 698. France.

Chauchard, P. (1970b). Le système nerveux, Presses Universitaires de France. Que sais-je? No. 8. France.

Chomsky, N. (2006). Language and Mind. Cambridge, MA: Cambridge University Press. https://doi.org/10.1017/CBO9780511791222

Cornille, P. (2003). Advanced Electromagnetism and Vacuum Physics. Singapore: World Scientific Publishing. https://doi.org/10.1142/5272

Dodson, F. (1971). How to Parent. USA: Signet Book.

Droit-Volet, S., \& Coull, J. (2015). The Developmental Emergence of the Mental Time-Line: Spatial and Numerical Distortion of Time Judgement. PLOS ONE, 10, e0130465.

https://journals.plos.org/plosone/article?id=10.1371/journal.pone.0130465 https://doi.org/10.1371/journal.pone.0130465

Eisberg, R., \& Resnick, R. (1985). Quantum Physics of Atoms, Molecules, Solids, Nuclei, and Particles (2nd ed.). New York: John Wiley \& Sons.

Fankel, T. (1997). The Geometry of Physics. Cambridge, MA: Cambridge University Press.

Flechsig, P. (1920). Anatomie des Menschlichen Gehirns und Rückenmarks auf Myelogenetischen Grundlage. Leipzig: Thienne.

Giancoli, D. C. (2008). Physics for Scientists \& Engineers. Upper Saddle River, NJ: Prentice Hall.

Goodfellow, I., Bengio, Y., \& Courville, A. (2016). Deep Learning. Cambridge, MA: MIT 
Press. http://www.deeplearningbook.org/

Griffiths, D. J. (1999). Introduction to Electrodynamics. Upper Saddle River, NJ: Prentice Hall.

Halgren, E. (1999). Emotional Neurophysiology of the Amygdala within the Context of Human Cognition. In J. P. Aggleton (Ed.), The Amygdala: Neurobiological Aspects of Emotion, Memory and Mental Dysfunction (pp. 191-228). New York: Wiley-Liss.

Hassani, S. (1999). Mathematical Physics. USA: Springer-Verlag.

Hawkins, J., \& Blakeslee, S. (2004). On Intelligence. New York: Owl Books.

Hebb, D. O. (1949). The Organization of Behavior. New York: Wiley.

Hibbeler, R. C. (2005). Mechanics of Materials. Upper Saddle River, NJ: Prentice Hall.

Howell, R. W., \& Bradley, W. J. (2001). Mathematics in a Postmodern Age. Grand Rapids, MI: William B. Eerdmans Publishing Company.

Jackson, J. D. (1999). Classical Electrodynamics. Hoboken, NJ: John Wiley \& Sons.

Lacy, J. W., \& Stark, E. L. (2013). The Neuroscience of Memory: Implications for the Courtroom. Nature Reviews Neuroscience, 14, 649-658.

https://www.nature.com/articles/nrn3563

https://doi.org/10.1038/nrn3563

Lide, D. R. (2003). CRC Handbook of Chemistry and Physics (84th ed.). New York: CRC Press.

Meriam, J. L., \& Kraige, L. G. (2003). Engineering Mechanics Dynamics. Hoboken, NJ: John Wiley \& Sons.

Michaud, A. (1997). Einstein's Operating System. Canada: SRP Books. https://www.smashwords.com/books/view/154227

Michaud, A. (2003). The Neurolinguistic Foundation of Intelligence. Canada: SRP Books. https://www.smashwords.com/books/view/156882

Michaud, A. (2013a). The Mechanics of Neutron and Proton Creation in the 3-Spaces Model. International Journal of Engineering Research and Development, 7, 29-53. http://ijerd.com/paper/vol7-issue9/E0709029053.pdf

Michaud, A. (2013b). Unifying All Classical Force Equation. International Journal of Engineering Research and Development, 6, 27-34. http://www.ijerd.com/paper/vol6-issue6/F06062734.pdf

Michaud, A. (2016a). Comprehension Process Overview. Journal of Biometrics \& Biostatistics, 7, Article ID: 1000317.

http://www.omicsonline.org/open-access/comprehension-process-overview-2155-6180 -1000317.pdf

Michaud, A. (2016b). Intelligence and Early Mastery of the Reading Skill. Journal of Biometrics \& Biostatistics, 7, Article ID: 1000327.

https://www.omicsonline.org/open-access/intelligence-and-early-mastery-of-the-readi ng-skill-2155-6180-1000327.pdf

Michaud, A. (2016c). On De Broglie's Double-Particle Photon Hypothesis. Journal of Physical Mathematics, 7, Article ID: 1000153.

https://www.omicsonline.com/open-access/on-de-broglies-doubleparticle-photon-hyp othesis-2090-0902-1000153.pdf

Michaud, A. (2017a). Electromagnetic Mechanics of Elementary Particles (2nd ed.). Saarbrücken, Germany: Scholar's Press.

https://www.morebooks.de/store/gb/book/electromagnetic-mechanics-of-elementary-p articles/isbn/978-3-330-65345-0 
Michaud, A. (2017b). On the Relation between the Comprehension Ability and the Neocortex Verbal Areas. Journal of Biometrics \& Biostatistics, 8, Article ID: 1000331. https://www.omicsonline.org/open-access/on-the-relation-between-the-comprehensio n-ability-and-the-neocortexverbal-areas-2155-6180-1000331.pdf

Michaud, A. (2017c). The Last Challenge of Modern Physics. Journal of Physical Mathematics, 8, Article ID: 1000217.

https://www.omicsonline.org/open-access/the-last-challenge-of-modern-physics-20900902-1000217.pdf

Michaud, A. (2018). The Hydrogen Atom Fundamental Resonance States. Journal of Modern Physics, 9, 1052-1110. https://file.scirp.org/pdf/JMP_2018042716061246.pdf https://doi.org/10.4236/jmp.2018.95067

Peterson, J. B. (1999). Maps of Meaning. New York: Routledge.

Piaget, J. (1974). The Origins of Intelligence in Children. Madison, CT: International Universities Press.

Piaget, J. (2001). The Language and Thought of the Child. London: Routledge \& Kegan.

Pickenhain, L. (1998). Das Schicksal der PARLOW schen Ideen in der UdSSR. In I. P. Pawlow (Ed.), Gesammelte Werke über die Physiologie und Pathologie der höhere Nerventätigkeit (pp. 375-408). Germany: Ergon Verlag.

Rao, S. S. (2005). Mechanical Vibrations. Singapore: Pearson Prentice Hall.

Sears, W., Zemansky, M. W., \& Young, H. D. (1982). University Physics. Boston, MA: Addison-Wesley.

Shepherd, G. M. (1994). Neurobiology (3rd ed.). New York: Oxford University Press.

Starr, A., Libertus, M. E., \& Brannon, E. M. (2013). Number Sense in Infancy Predicts Mathematical Abilities in Childhood. Proceedings of the National Academy of Sciences of the United States of America, 110, 18116-18120.

http://www.pnas.org/content/pnas/110/45/18116.full.pdf https://doi.org/10.1073/pnas.1302751110

Van Petten, C., \& Luka, B. (2006). Neural Localization of Semantic Context eVects in Electromagnetic and Hemodynamic Studies. Brain and Language, 97, 279-293. 